IVAN CÉSAR RIBEIRO

\title{
REGULAÇÃO FINANCEIRA, PODER NO MERCADO E CRISE FINANCEIRA
}

TESE DE DOUTORADO

Orientador: Professor Doutor Calixto Salomão Filho

FACULDADE DE DIREITO

SÃO PAULO

2012 
Prof. Dr. João Grandino Rodas Reitor da Universidade de São Paulo

Prof. Dr. Antonio Magalhães Gomes Filho Diretor da Faculdade de Direito

Prof. Dr. Paula Andrea Forgioni

Chefe do Departamento de Direito Comercial

Profa. Dra. Monica Herman Salem Caggiano

Presidente da Comissão de Pós-Graduação em Direito 


\title{
REGULAÇÃO FINANCEIRA, PODER NO MERCADO E CRISE FINANCEIRA
}

\author{
TESE DE DOUTORADO
}

Orientador: Professor Doutor Calixto Salomão Filho

Tese apresentada à Banca Examinadora da Faculdade de Direito da Universidade de São Paulo, como requisito para a obtenção do título de Doutor em Direito, sob a orientação do Professor Doutor Calixto Salomão Filho.

FACULDADE DE DIREITO

SÃO PAULO

2012 
- ... Et le génie exige la patience à travailler, docteur, et plus je vais, citoyen, moins je crois à l'efficacité des soudaines illuminations qui ne seraient pas accompagnées ou soutenues par un travail sérieux, moins je crois à l'efficacité des conversions extraordinaires soudaines et merveilleuses, à l'efficacité des passions soudaines, - et plus je crois à l'efficacité du travail modeste, lent, moléculaire, définitif.

Charles Péguy, Encore de la grippe, Cahiers de la quinzaine, vol. I, $\mathrm{n}^{\circ}$ 6, 20 de março de 1900. 
Aos meus pais, Marlene e Antônio. 


\section{Agradecimentos}

O grande exercício que se faz ao escrever uma tese de doutorado é o de humildade, e esse exercício começa pelo reconhecimento de que uma tese não se faz sozinho, são muitas as ajudas e contribuições que se recebe ao longo do caminho.

O primeiro agradecimento vai ao professor Calixto Salomão Filho, por aceitar realizar essa orientação e por todo o trabalho envolvido na produção da tese. Orientar um neófito nesta seara do Direito Concorrencial, depois de já ter escrito sua doutrina fundamental e ter avançado para pautas de pesquisa mais importantes é uma mostra de grande desprendimento e generosidade, para a qual nenhum agradecimento é suficiente.

Agradeço também ao professor Denisard Alves, membro da banca de qualificação, além de ter sido meu orientador em meu primeiro mestrado. Devo a ele não só os alicerces da econometria (aliás, jurimetria, já que há muitos anos o professor Denisard me apresenta como jurimetrista), mas também as indicações de referências fundamentais da pesquisa que aqui apresento.

Agradeço ao professor Gilberto Bercovici, que também participou da banca de qualificação. Sempre que pudemos conversar, chamou-me o professor Gilberto a atenção para a importância de considerar a relação do tema com o desenvolvimento econômico, com a inovação e com a atuação do Estado, algo que espero ter conseguido abordar de forma suficiente no trabalho.

Com o professor João Manoel Pinho de Mello tive o prazer compartilhar discussões não apenas sobre economia bancária, mas também sobre a economia do crime, dois temas que nos interessam profundamente. $\mathrm{O}$ professor João de Mello é, sem dúvida nenhuma, um dos grandes pesquisadores em regulação bancária, e é um privilégio e uma honra poder receber seus comentários neste trabalho tão despretensioso. 
Comecei a desenvolver a presente pesquisa ainda durante minha temporada na Universidade de Yale, cursando meu segundo mestrado. Uma trágica, mas proveitosa coincidência, fez com que meu período naquela Universidade coincidisse com o ápice da Crise de 2008. Agradeço a acolhida e generosidade do professor Robert Shiller, sem dúvida uma referência fundamental ao analisar as crises e a regulação financeira.

Ainda em referência a Yale, agradeço as discussões e a boa vontade de Michael Solender, que, depois de trabalhar como diretor jurídico do Bear Sterns e do Washington Mutual, ofereceu a disciplina Anatomy of the Financial Crisis na Faculdade de Direito. Ficou na minha memória a paciência e dedicação com que recebia este único aluno estrangeiro em meio a uma dúzia de estudantes da matéria. Também agradeço as discussões, durante o curso e fora dele, com Gregory Flemming (Merrill Lynch) e Alan Schwartz (Bear Sterns).

As discussões sobre o projeto de pesquisa com a professora Roberta Romano, além daquelas em seu curso Contemporary Issues in Law and Business, foram muito importantes para o trabalho, assim como as discussões sobre regulação com a professora Susan Rose-Ackerman e sobre modelos econométricos para eventos raros com o professor John Donohue.

Agradeço ao professor Ernesto Zedillo, pelas discussões sobre as crises financeiras na América Latina e pela oportunidade de participar da Conferência sobre a Crise Financeira, organizada pelo Yale Center for Studies of Globalization. Foi de inestimável ajuda a oportunidade de discutir com Raghuram Rajan, Carmen Reinhart, Viral Acharya e outros, naquele evento.

Agradeço a oportunidade de poder discutir e refinar as ideias deste trabalho que me proporcionaram Demosthenes Pinho Neto (Itaú-Unibanco), Jair Ribeiro (Indusval), Pedro Bastos (HSBC), além de todos os que me receberam diversas vezes na Anbima, aos quais agradeço na pessoa de Eurídson Sá. Agradeço ao professor Jairo Saddi por discussões específicas sobre a querela entre o CADE e o Bacen, bem como a todos no Banco Central, 
em especial o colega de turma Jeffeson Alvares, além de Isaac Menezes, Adalberto Rocha, Cristiano Cozer, Ailton dos Santos, Walkyria de Oliveira, Marcel dos Santos e Alexandre Maia.

Agradeço ao professor Marcos Paulo Verissímo, conselheiro do CADE, pela discussão sobre aspectos de Direito Administrativo do caso CADE vs. BACEN, e ao professor Márcio Nakane pelas discussões sobre as relações entre regulação bancária e concorrência no pós Crise de 2008. As discussões com professor Simone Sepe, da Universidade do Arizona, foram essenciais para tornar mais claras algumas das idéias no trabalho e em especial para refinar o resumo na versão em italiano, onde também pude contar com a ajuda de Andrea Bertolini, colega em minha estada no programa de mestrado em direito da Universidade de Yale.

Contribuições importantes para este trabalho vieram das discussões sobre mercado de capitais, em especial sobre a regulação de fundos de investimento, sobre fundos imobiliários e sobre a análise de custos e benefícios da regulação. Agradeço em especial a Luciana Dias, Maria Helena Santana e Otávio Yazbek. Ao Otávio agradeço também pelas discussões específicas sobre a regulação financeira e as referências de grande valia que me indicou, e mesmo me emprestou. Ainda na CVM foram inestimáveis as contribuições de Flávia Mouta, Guilherme Parente, Cláudio Maes, Francisco Bastos Santos e Roberto Tadeu Fernandes, entre muitos que me ajudaram naquela autarquia.

Ainda da indústria de fundos, agradeço as discussões com Carlos Ambrósio (Claritas), Regis Abreu (Mercatto), Luis Eugênio Figueiredo (ABVCap e Rio Bravo), Zeca Oliveira (BNY Mellon), Sérgio Beleza, Rodrigo Machado (Brazilian Mortgages) e Carlos Rebello (Bovespa).

Em minha família, além do apoio e carinho de todos, agradeço ao meu irmão Wladimir, cuja diligência em ligar para falar de referências, e pelos incontáveis livros e materiais que me trouxe, fariam pensar que seria ele a escrever a tese. Ao Alex agradeço pelo apoio na preparação do texto, afinal não é sempre que se conta com um Prêmio Jabuti para opinar sobre estilo e 
qualidade da escrita, também agradeço a Márcia Pescuma que reviu o texto e a Beatriz Barros pela confecção de maior parte das tabelas do trabalho.

Agradeço às bibliotecárias e aos blibiotecários da Faculdade de Direito, da FEA e de Yale de ajuda inestimável durante todo o período da pesquisa, agradecimento que faço nas pessoas de Raquel Lima, Raquel Silveira e Roberto Klinguelfus. Muito de uma tese depende também do trabalho da seção de pós-graduação. Agradeço em especial a Fátima Cortinhal e a Amanda Ferrari. Também agradeço a Daniella Omoto, que lidou com muitas das solicitações de última hora típicas de um pós-graduando.

São muitos os que leram, fizeram sugestões e que ofereceram toda forma de ajuda na preparação do projeto que precedeu a tese e do texto do exame de qualificação. Por essa ajuda agradeço a Dennis David, Priscilla César, Brisa Ferrão, Caroline Gross, Henry Hansmann, Tafari Lumumba, Paul Rodriguez, Robert Wai, com a certeza de que cometo alguma injustiça nesses agradecimentos ao esquecer algum nome.

Chega-se ao final de um programa de doutorado com muito mais dúvidas e muito menos certezas do que no começo. A cada dia de estudo e de pesquisas, esvaem-se todas aquelas ideias que pareciam originais e os sonhos de reinventar a ciência. A contribuição que se faz será sempre pequena, ainda que com boa vontade possa ser dita original, e não virá sem muito, mas muito trabalho e esforço. Nesse sentido me pareceu bastante apropriada a citação que escolhi para a abertura deste trabalho, na versão original de Peguy, que me parece muito mais apropriada que a de Oliver Williamson, que foi de quem primeiro a escutei:

"Quanto mais eu vivo menos acredito em iluminações súbitas, que não venham acompanhadas por trabalho sério. Menos eu creio nas súbitas paixões e mais eu creio na eficiência do trabalho modesto, lento, molecular e definitivo." 


\section{Resumo}

Os bancos nunca foram tão grandes como depois da Crise de 2008. No momento de maior pânico, logo após a quebra do Lehman Brothers, autoridades do mundo inteiro autorizaram fusões e aquisições antes vetadas. Era preciso garantir a estabilidade do sistema financeiro - alegava-se - e tentar preservar a concorrência nesse instante apenas aumentaria o pânico.

O Brasil não ficou imune a esse movimento. Fusões como a do Itaú com o Unibanco e aquisições como a da Nossa Caixa pelo Banco do Brasil levaram o setor a um grau de concentração nunca visto antes. A discussão entre o Conselho Administrativo de Defesa Econômica (CADE) e o Banco Central, sobre quem deve julgar tais concentrações, faz parecer que existe uma contradição entre a disciplina constitucional da defesa da concorrência e a garantia da segurança e estabilidade das instituições financeiras. O resultado é a proliferação de instituições hipertrofiadas, os megabancos, em prejuízo desses mesmos princípios da ordem concorrencial estabelecidos constitucionalmente.

Os principais argumentos em favor dos megabancos seriam, primeiro, o de que as rendas derivadas de poder no mercado que estes auferem (o chamado valor de franquia) formaria um colchão que aumentaria a sua resistência no caso de choques como o de 2008. Em segundo lugar, sugere-se que esses bancos, ao crescerem, acumulariam ganhos de escala, de escopo e de eficiência custo.

Este trabalho propõe que não existe nenhum antagonismo entre a defesa da concorrência e a regulação bancária tradicional, de cunho prudencial e sistêmico. Propõe ainda que o modelo dos megabancos coloca um grande risco para a sociedade, tratando-se na realidade de um movimento estratégico de grandes instituições para acumular mais poder no mercado. São dois os motivos pelos quais se defende que não existe nenhum ganho no crescimento dessas instituições. 
Em primeiro lugar, as economias de escala se esgotam muito cedo, proposição com amplo suporte teórico e empírico. Na previsão mais otimista, bancos com mais do que 25 bilhões de dólares em ativos já estão na área de deseconomias de escala. Tampouco existem economias de escopo que autorizem a concentração de atividades tão diversas como as de banco comercial e de investimento. Bancos que concentram muitas atividades são, na realidade, avaliados negativamente pelo mercado. Mesmo os ganhos de eficiência custo, resultantes de uma melhor gestão de instituições mal administradas, não tem suporte empírico relevante.

Em segundo lugar, uma estrutura moderna do setor bancário pressupõe bancos especializados e concentrados nas áreas em que têm maior eficiência. São bancos menores, que dividem com os mercados financeiros e outros intermediários a tarefa de prover o crédito. A concorrência do mercado de capitais, de instituições não bancárias (como gestores de fundos e financeiras) e de instituições não financeiras (como redes de supermercados, correios e empresas comerciais) forçou esses bancos a fazer o descruzamento de subsídios e a abandonar as atividades em que eram menos eficientes. Os megabancos vão na contramão dessa modernização, negando os princípios da Ordem Concorrencial.

A reação dessas instituições, entretanto, é contundente. Os bancos procuram o crescimento excessivo, de forma a criar as megainstituições, para colher ganhos que não vêm de uma operação mais eficiente. São ganhos provindos das inconsistências na atuação do regulador. Este trabalho propõe a extensão das doutrinas de comportamento estratégico, de forma a incluir três categorias novas de comportamentos adotados pelos megabancos:

1. Expansão Não-Eficiente de Participação no Mercado: Bancos operam muito além da escala eficiente para obter as vantagens da garantia de socorro aos grandes bancos (o too big to fail), para influenciar a regulação e aumentar lucros e, por fim, para explorar os acionistas não controladores.

2. Saturação Anticompetitiva de Mercados: Bancos acumulam produtos para além do recomendado pelos ganhos de escopo, e também agências além do que geraria ganhos de escala, para bloquear a entrada de novos concorrentes. Mostra-se neste trabalho como o excesso de agências e produtos funciona como uma barreira à entrada, o que explicaria essas expansões como um movimento preventivo. 
3. Bloqueio de Modernização Pró-Competitiva: Como uma estrutura moderna do setor obriga uma redução do tamanho dos bancos e, também, uma redução da participação do setor bancário nas atividades de crédito, os bancos tentam bloquear a modernização. O bloqueio é feito através de práticas anticoncorrenciais já conhecidas, como o bloqueio ao acesso de bens essenciais (por exemplo, ao sistema de pagamentos) e as ações concertadas, entre outros.

A resposta do regulador para esses comportamentos estratégicos seria a aplicação pura e simples das ferramentas do Direito Concorrencial. Este deve aplicar medidas ordenando a desconcentração de mercados e deve investigar e punir as práticas anticompetitivas. É uma atuação que difere, portanto, da regulação bancária tradicional, em que constantemente se consideram os aspectos prudenciais e sistêmicos. Isso ocorre porque, no caso desses comportamentos, o restabelecimento da livre concorrência é condição necessária e suficiente para garantir a segurança e a higidez dos mercados financeiros.

Essa conclusão, aplicada ao Brasil, leva a que se deve proceder à desconcentração no setor, com a adoção de medidas compensatórias para a maioria das fusões recentemente aprovadas. Essas medidas encontram precedente significativo naquelas adotadas tanto na Europa quanto nos Estados Unidos durante a Crise de 2008.

Finalmente, algumas das previsões das hipóteses desenvolvidas no trabalho são testadas empiricamente. Foi desenvolvido um modelo jurimétrico que mostra que mais competição resulta em maior estabilidade financeira. $\mathrm{O}$ modelo também confronta a abordagem da Nova Economia Institucional com a NeoEstruturalista, mostrando que esta última resulta em mais competição e maior estabilidade financeira.

Palavras-chave: Sistema Financeiro, Concorrência Bancária, Estrutura de Mercado.

Códigos JEL: G18, G21, L13, L16 


\section{Abstract}

Banks have never been as great as after the 2008 crisis. At the moment of greatest panic, just after the collapse of Lehman Brothers, authorities all around the world authorized otherwise unlawful mergers and acquisitions. It was necessary to ensure the stability of the financial system, it was claimed, and try to preserve competition right now would only increase the panic.

Brazil has not been immune to this trend. Mergers such as Itaú and Unibanco and operations as the acquisition of Nossa Caixa by Bank of Brazil led the industry to a concentration degree never seen before. The discussion between the Council for Economic Defense (CADE) and the Brazilian Central Bank, about who should examine such concentrations, makes it appear that there is a contradiction between the constitutional underpinnings of antitrust policy and the ensuring of soundness and stability of financial institutions. The result is a proliferation of institutions hypertrophied, the megabanks, with unrepairable damages to the principles of competition constitutionally assured.

The main arguments in favor of megabanks would be, first, that the income derived from market power they earn (franchise value) form a buffer that increases its resistance to such shocks as the 2008 Crisis. Secondly, it is suggested that banks accumulate economies of scale, scope and cost efficiency as they grow.

This research proposes that there is no antagonism between antitrust law and traditiona banking regulation, more focused in prudential and systemic aspects. It also proposes the model of megabanks poses a major risk to society, since it is actually a strategic move from large institutions to accumulate more market power. There are two reasons why it is argued that there is no gain in the growth of these institutions.

First, economies of scale are exhausted too early, a proposition that rests in extensive theoretical and empirical support. In the most optimistic forecast, banks with more than $\$ 25$ billion in assets are already incurring in scale 
diseconomies. Nor are there economies of scope allowing the concentration of activities as diverse as commercial and investment banking. Financial institutions that concentrate many activities are actually evaluated negatively by the market. Even the cost efficiency gains resulting from better management of institutions has no relevant empirical support.

Second, a modern financial system requires specialized banks, focused in areas which they have greater efficiency. They are smaller banks, which share with the financial markets and other intermediaries the task of providing credit. The competition provided by non-bank institutions (such as mutual funds and credit unions) and non-financial institutions (such as retail stores and conglomerates) forced these banks to do the unwinding of subsidies and abandon activities they were less efficient. Megabanks go against this modernization, and they are a deny of the principles of the competition order.

The reaction of these institutions, however, is striking. Banks seek overgrowth in order to create megainstitutions, seizing profits that does not come from a more efficient operation. They seek gains stemmed from inconsistencies in the work of regulators. This work suggests the extension of the opportunistic behavior doctrines, in a way to include three new types of strategic behavior adopted by the megabanks:

1. Non-efficient Increase of Market Share: Banks operate far beyond efficient scale to take advantage of the implicit government guarantee of the rescuing of large banks (the too big to fail policy), to influence regulation and thus increase profits, and finally, to explore noncontrolling shareholders.

2. Anticompetitive Market Crowding: Banks accumulate products beyond what is recommended in order to attain gains of scope, and also agencies in excess of what generates economies of scale, doing so to block the entry of new competitors. It is shown here how the excess branches and products acts as entry barrier, explaining these expansions as a preemptive move.

3. Blocking of Pro-Competitive Modernization: As a modern industry structure requires a reduction in the size of banks and also a reduction in the share of the banking sector in lending activities, banks try to block the modernization. This blocking is done through anticompetitive practices already known, such as denying access to essential facilities (eg, the payment system) and by adopting a collusive behavior, among others. 
The answer of regulators for such strategic behavior would be a pure and simple application of Competition Law remedies. They should apply measures ordering the deconcentration of markets and should investigate and punish anticompetitive practices. This approach differs from traditional banking regulation, in which constantly consideration of prudential and systemic aspects reign. This is because, in the case of these behaviors, restoring free competition is necessary and sufficient condition to ensure the safety and soundness of financial markets.

This conclusion applied to Brazil, means that one must increase competition in the industry, with the adoption of compensatory measures to the most recently approved mergers. These measures have a significant precedent in the measures adopted in both Europe and the United States during the Crisis of 2008.

Finally, some of the predictions of these hypotheses are tested empirically. It is developed a jurimetric model, which shows that more competition yields more financial stability. The model also confronts the New Insitutional Economics approach to the question with a neo-structuralist approach, showing that the former entails more competition and financial stability.

Keywords: Financial System, Bank Competition, Market Structure.

JEL Code: G18, G21, L13, L16 


\section{Sinossi}

Le banche non sono mai state così grandi come dopo La crisi Del 2008. Nel momento di pânico maggiore Del mercato, subito dopo il crollo di Lehman Brothers, le autorità di tutto hanno autorizzato fusioni e acquisizioni che in altri tempi sarebbero state considerate in violazione di norme imperative. $\grave{E}$ stato affermato Che tale fratura con l'ordinamento sia stata necessaria per assicurare la stabilità del sistema finanziario. Invero, preservare la concorrenza in quel determinato momento avrebbe soltanto aumentato il pânico dei mercati.

Il Brasile non è stato immune da questa tendenza. Fusioni come Itaú e Unibanco e operazioni come l'acquisizione di Nossa Caixa dalla Banca Del Brasile hanno determinato un livello di concentrazione nel settore finanziario senza precedenti. La discussione tra Il Consiglio per la difesa economica (CADE) e la Banca Centrale del Brasile circa la competenza di verificare gli effetti delle concentrazioni, evidenzia una contraddizione tra le basi costituzionali della politica antitrust e l'obiettivo di garantire la solidità e stabilità delle istituzioni finanziarie. Il risultato è stato la proliferazione di istituzioni finanziarie ipertrofiche, le megabanche, con conseguenti danni irreparabili ala concorrenza, principio costituzionalmente farantito.

Gli argomenti principali a favore delle megabanche sarebbero i seguenti: (i) i proventi derivanti da un potere di mercato determinerebbero un aumento Del capitale econômico in grado di meglio neutralizzare le conseguenze avverse della crisi del 2008; e (ii) al crescere delle banche l'effcienza allocativa aumenterebbe sulla base di economie di scala.

Questa ricerca sostiene Che non vi sarebbe antagonismo tra il diritto antitrust e gli obiettivi tradizionali della regolazione bancaria mirati, appunto, al controllo prudenziale delle istituzioni finanziarie e ai conseguenti effetti sistemici. Tale ricerca suggerisce che il modello di megabanche rappresenta un grave rischio per la collettività, in quanto favorirebbe grandi istituzioni nell'accumulare strategicamente potere di mercato. Pertanto non vi sarebbe nessun guadagno di efficienza nel favorire tali concentrazioni. Nella presente ricerca si offrono due motivazioni.

In primo luogo, come ampiamente supportato teoricamente e empiricamente, le economie di scala si esauriscono nel breve periodo. Nella previsione più ottimistica, le banche con più di \$25 miliardi di dollari in attività starebbero, 
invero, già operando in diseconomie di scala. Inoltre, non vi sarebbero economie di scopo che consentono la concentrazione di attività diverse come banche commerciali e di investimento. A ciò si aggiunga che le istituzioni finanziarie che concentrano molte attività sono valutate negativamente dal mercato. Infine i la riduzione dei costi derivanti dall'aumento della scala sarebbe privo di supporto empirico.

In secondo luogo, un moderno sistema finanziario impone alle banche specializzate di operare in quei settori dove l'effcienza produtiva individuale è maggiore. Queste banche di minore dimensione hanno il compito di fornire il credito congiuntamente ai mercati finanziari ed a altre istituzioni finanziarie (non bancarie). La competizione di istituzioni non bancarie (come fondi comuni di investimento e cooperative di credito) e non finanziarie (come, ad esempio, distribuzioni al dettaglio e all'ingrosso) costringono le banche ad abbandonare tutte quelle attività meno efficienti. Le Megabanche, peranto, andrebbero contro questa tendenza di modernizzazione del settore, negando, tra l'altro, i principi basilari della concorrenza.

La reazione delle banche, tuttavia, è impressionante. Invero, cercando una crescita eccessiva al fine di creare megainstitutioni, le banche percepiscono rendite da concentrazione, ottenendo, tra l'altro, extra-guadagni derivanti dalle incongruenze del sistema di regolamentazione. Questo lavoro, peranto, suggerisce l'estensione della dottrina sui comportamenti opportunistici, in modo tale da includere tre nuovi tipi di comportamento strategico adottato dalle megabanche:

1. Non efficiente aumento della quota di mercato: banche Che operano ben al di là del livello di efficienza per sfruttare la garanzia implicita di salvataggio che il governo appresta alle banche di grandi dimensioni (la garanzia alle cc.dd. istituzioni too big to fail). In tal modo le banche sarebbero ingrado di aumentare i profitti, e, infine, per esplorare azionisti di minoranza.

2. "Affollamento Anticoncorrenziale": banche Che offrono prodotti al di là di ciò che l'efficienza raccomandere. Inoltre, banche si articolano in "sportelli" al di sopra del livello ottimale suggerito dalle economie di scala in modo di bloccare l'ingresso di nuovi concorrenti. In questo caso, gli sportelli e i prodotti in eccesso agirebbero quale barriera all'ingresso e, per l'effetto, l'espansione dimensionale dell'istituzione finanziaria sarebbe una scelta strategica preventiva.

3. "Blocco della Modernizzazione Competitiva": Poichè una moderna struttura industriale richiederebbe una riduzione delle dimensioni delle banche e della quota del settore bancario in attività di prestito, le banche cercano strategicamente di bloccare il processo di modernizzazione. Questo "bloco" si attuerebbe tramite pratiche anticoncorrenziali note, come negare l'accesso ai servizi essenziali (ad esempio, i sistemi di pagamento) e con l'adozione di comportamenti collusivi. 
La risposta delle autorità di regolamentazione a tali comportamenti strategici dovrebbe essere una semplice applicazione dei rimedi di diritto della concorrenza. Semplicemente: Le autorità dovrebbero ordinare la deconcentrazione del mercato nonchè indagare e sanzionare le pratiche anticoncorrenziali. Questo approccio differisce dalla regolamentazione bancaria tradizionale, dove considerazioni di vigilanza prudenziale e stabilità sistêmica dominano Il dibattito. In ultima analisi si ritiene in questo Studio Che ripristinare la libera concorrenza sia condizione necessaria e sufficiente per garantire la sicurezza e la solidità dei mercati finanziari.

Applicare queste conclusioni in Brasile significa aumentare la concorrenza nel settore, con l'adozione di misure compensative per le concentrazioni più recentemente approvato. Queste misure hanno un precedente significativo nelle misure adottate in Europa e negli Stati Uniti durante la crisi del 2008.

Infine, alcune delle queste ipotesi suggerite in questo Studio sono testate empiricamente. Sviluppando un modelo jurimetric, si mostra come al crescere della concorrenza creasca anche la stabilità finanziaria. Il modello si confronta anche pone anche di fronte La Nuova economia Istituzionali affrontare La questione con un approccio neo-strutturalista, che mostra che il primo implica una maggiore concorrenza e la stabilità finanziaria.

Parole chiave: Sistema finanziari, la concorrenza bancaria, struttura del mercato.

JEL Codice: G18, G21, L13, L16 


\section{Sumário}

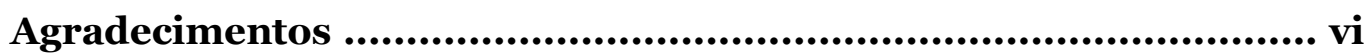

Resumo

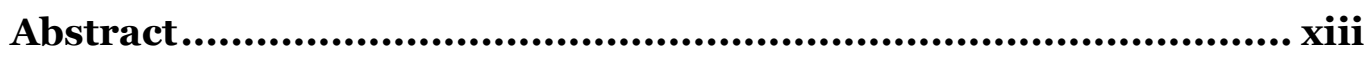

Sinossi

Lista de Tabelas e Gráficos ............................................................xxv

Parte I ...............................................................................................1

Introdução ...................................................................................................1

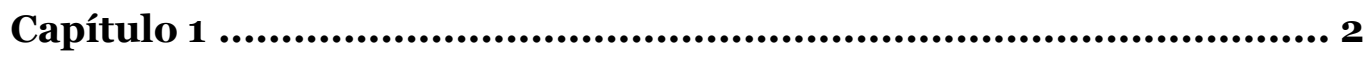

Introdução …............................................................................................. 2

1.1 A Crise de 2007/2008 ………………………………………………………4

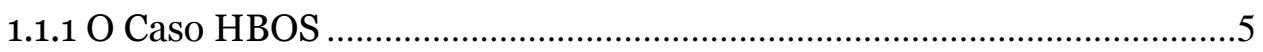

1.1.2 Outras Decisões da Comissão Europeia...................................................9

1.1.3 Os EUA e a Concorrência Bancária …………………………………....12

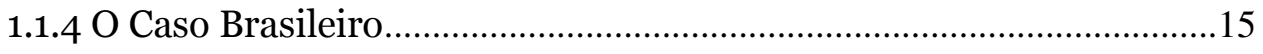

1.2 A Evolução da Regulação Financeira ………………………………………....20

1.3 Hipóteses e Desenvolvimento do Trabalho .......................................................21

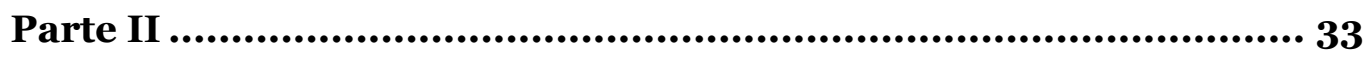

As Falhas Fundamentais e a Regulação Financeira.......................... 33

Capítulo 2 ..................................................................................... 34

Falhas da Intermediação Financeira ............................................. 34

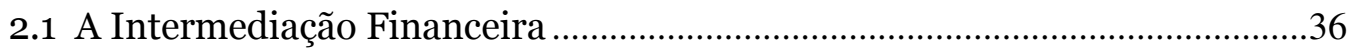

2.1.1 A Transformação de Ativos....................................................................37

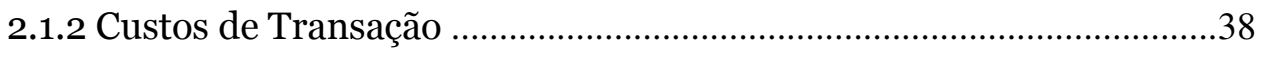

2.1.3 Assimetrias de Informação .................................................................42 


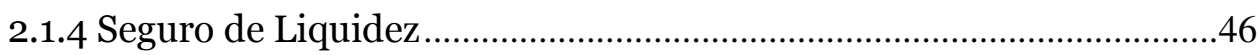

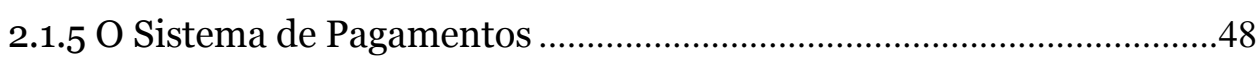

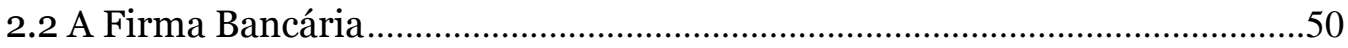

2.2.1 A Concorrência Perfeita.............................................................................51

2.2.2 O Modelo de Monti-Klein para Monopólio ............................................52

2.2.3 A Concorrência Oligopolística...............................................................53

2.2.4 Competição Monopolística e Oligopólio................................................54

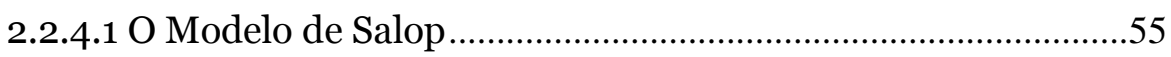

2.2.4.2 O Modelo de Fudenberg-Tirole-Eaton-Lipsey .........................58

2.2.4.3 Implicações Empíricas do Trade Diversion Effect..................63

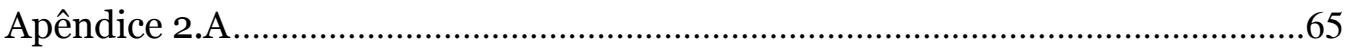

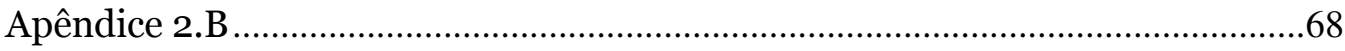

Capítulo 3 [...................................................................... 71

Falhas e Incentivos da Regulação Financeira............................. .71

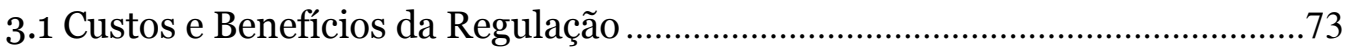

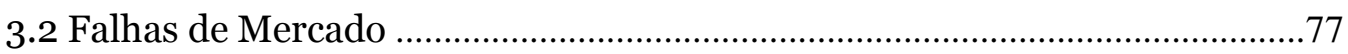

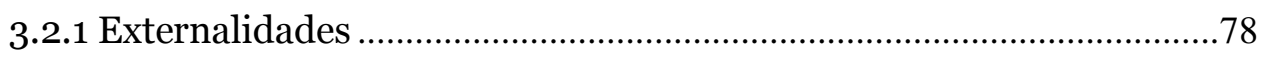

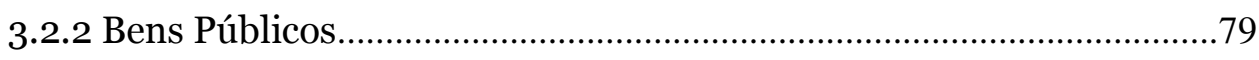

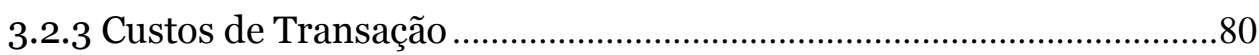

3.2.4 A Assimetria de Informações, o Risco Moral e a Seleção

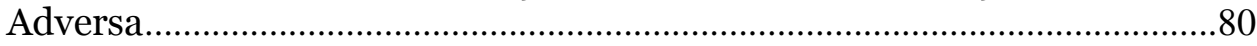

3.2.5 Conflito entre Agente e Principal.........................................................81

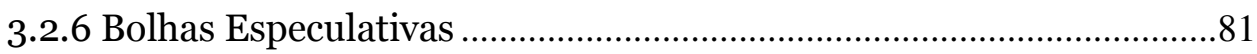

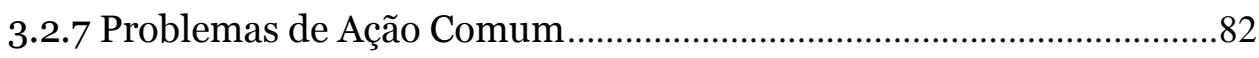

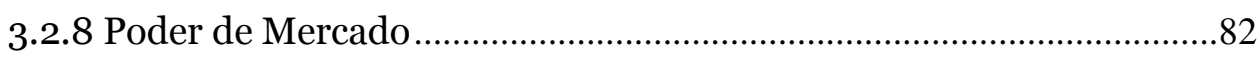

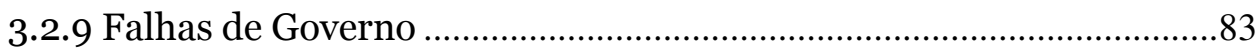

3.3 Os Modelos Microeconômicos de Regulação.........................................................83

3.3.1 O Modelo de BRYANT-DIAMOND-DYBVIG .............................................85

3.3.1.1 O Equilíbrio no Regime de Autarquia .......................................87

3.3.1.2 O Equilíbrio com um Mercado Financeiro................................8

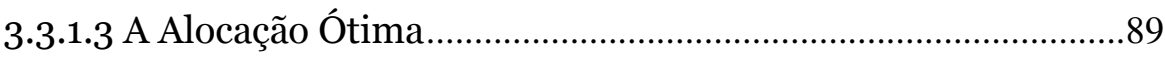


3.3.2 Os Equilíbrios com a Intermediação de Bancos ...................................91

3.3.2.1 Redução dos Colaterais ...........................................................93

3.3.2.2 Mudança de Expectativas.........................................................93

3.3.2.3 As Manchas Solares...................................................................95

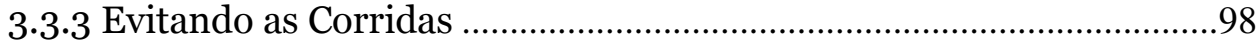

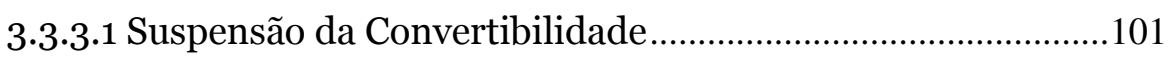

3.3.3.2 O Socorro Financeiro .................................................................103

3.3.3.3 As Câmaras de Compensação e Liquidaçãa............................106

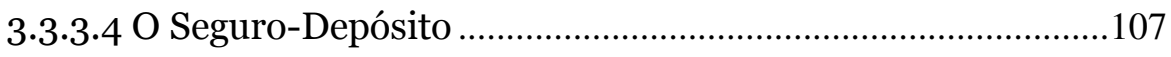

3.3.3.5 Outras Soluções ......................................................................108

3.3.4 Os Incentivos Para a Tomada de Risco...............................................109

3.3.5 O Valor de Franquia e o Papel do Capital...........................................116

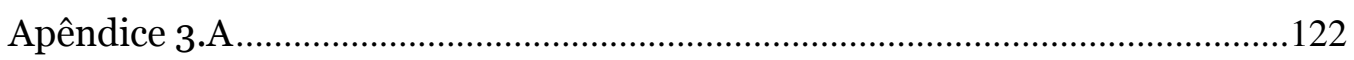

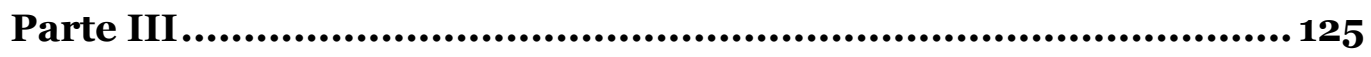

Regulação e Desregulação Financeira em Espécie ....................... 125

Capítulo 4 [...................................................................................126

A Regulação em Espécie e Incentivos Decorrentes .......................126

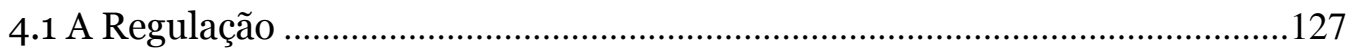

4.1.1 Algumas Classificações da Regulação ...................................................129

4.1.2 As Regulações em Espécie.................................................................131

4.1.2.1 Restrição de Entrada e Autorizações .......................................133

4.1.2.2 Estabelecimento de Agências ....................................................134

4.1.2.3 Restrição às Fusões e aos Conglomerados .............................135

4.1.2.4 Separação de Atividades Comerciais........................................136

4.1.2.5 Seguro de Depósito .................................................................137

4.1.2.6 Teto para Juros em Depósitos.................................................139

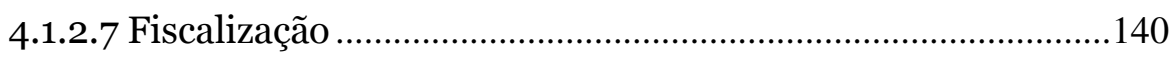

4.1.2.8 Exigências de Capital .............................................................141

4.1.2.9 Contabilidade a Valores de Mercado.......................................143 
4.1.2.10 Limites de Exposição a um Único Tomador .143

4.1.2.11 Proteção ao Consumidor ............................................................144

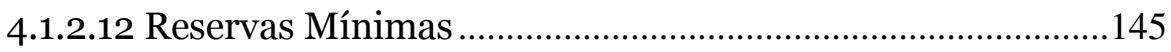

4.1.2.13 Janela de Redesconto .............................................................146

4.1.2.14 Liquidação de Instituições .......................................................148

4.1.3 Classificação Dialética da Regulação Financeira ..............................150

4.1.3.1 A Regulação Primária ............................................................152

4.1.3.2 A Regulação Secundária.........................................................156

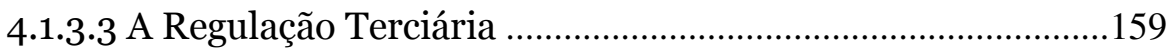

Capítulo 5 (.........................................................................164

Desregulação, Concorrência e Estabilidade.................................164

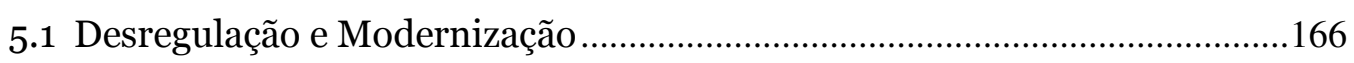

5.1.1 Desmembramento de Funções e a Especialização ..............................167

5.1.2 Desregulação e Aumento da Competição ...........................................170

5.1.3 Desintermediação..............................................................................173

5.1.4 A Modernização dos Bancos .............................................................176

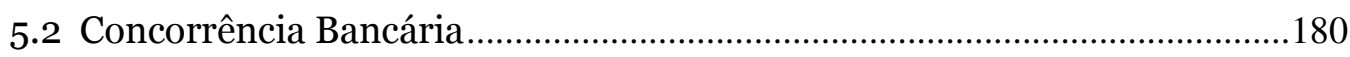

5.2.1 Evidência Empírica sobre a Concorrência .........................................186

5.2.2 A Concorrência Bancária no Brasil.....................................................188

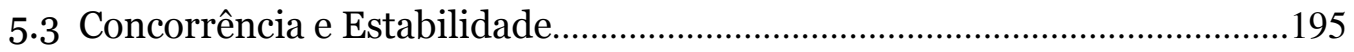

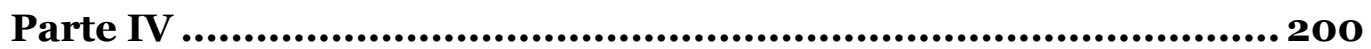

Comportamentos Estratégicos e Conclusões ................................200

Capítulo 6 ...............................................................................201

Regulação e Comportamentos Estratégicos ..................................201

6.1 Aumento Não Eficiente de Participação ........................................................206

6.1.1 Os Ganhos de Eficiência......................................................................207

6.1.2 Bancos Grandes Demais para Quebrar .............................................212

6.1.2.1 Origem e Desenvolvimento da Política de TBTF ..................213 


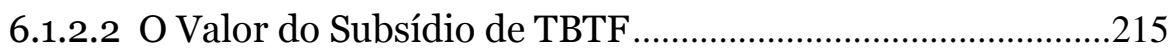

6.1.2.4 TBTF e Direito Concorrencial ..................................................222

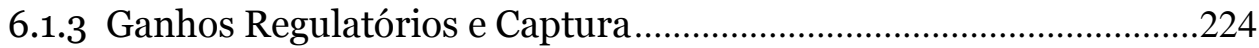

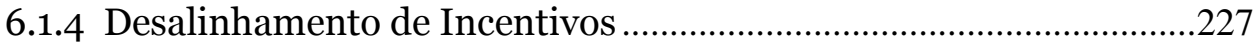

6.2 Saturação Anticompetitiva de Mercados..........................................................229

6.2.1 As Economias de Escala e Escopo.......................................................230

6.2.2 O Lançamento de Produtos ..................................................................232

6.2.3 A Abertura e Compra de Agências .....................................................235

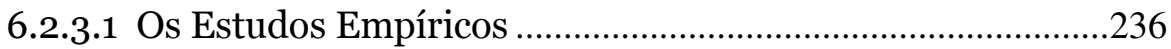

6.2.3.2 Agências como Barreira de Entrada ......................................237

6.2.4 A Saturação e o Direito Concorrencial .................................................239

6.3 Bloqueio de Modernização Pró-Competitiva...................................................242

6.3.1 O Sistema de Pagamentos como Essential Facility ..........................243

6.3.2 A Padronização de Tarifas ..................................................................245

Capítulo 7 ...................................................................................252

Testes Empíricos ........................................................................252

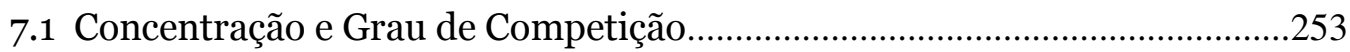

7.1.1 O Teste de SCHAECK - CIHAK - WOLFE...................................................256

7.1.2 Resultados para Concorrência e Concentração ...................................258

7.1.3 Resultados para Crise e Estrutura da Concorrência .........................269

7.1.4 Estrutura da Concorrência e Grau de Competição .............................277

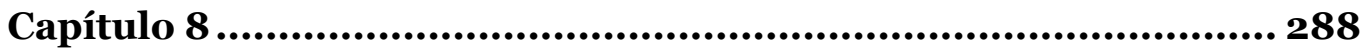

Conclusão - Fatiando os Megabancos ......................................... 288

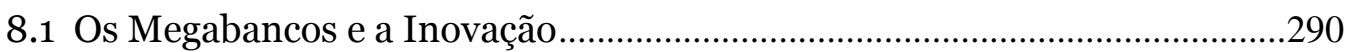

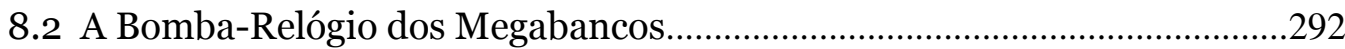

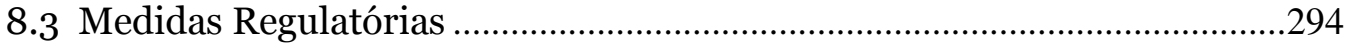

REFERÊNCIAS BIBLIOGRÁFICAS ................................................. 300

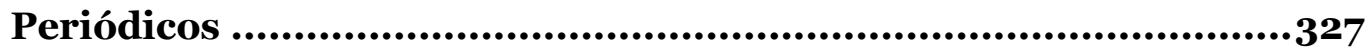




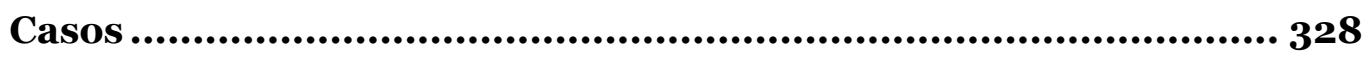

Regulações ............................................................................ 329 


\section{Lista de Tabelas e Gráficos}

\section{Tabelas}

Tabela 1.1 - Fusões e Aquisições (desde 1998 até 2012)..................................25

Tabela 1.2- HHI dual em termos de ativos por país...........................................31

Tabela 1.3- HHI dual em termos de empréstimos, por país................................32

Tabela 3.1 - Recomendações da OCDE........................................................74

Tabela 3.2 - Falhas de Mercado....................................................................78

Tabela 3.3- A Relação entre o Momento de Maiores Mudanças.......................100

Tabela 3.4 - Payoffs do Seguro Depósito..........................................................111

Tabela 3.5 - Frequência de Crises Bancárias: África e Ásia até 2008...............122

Tabela 3.6 - Frequência de Crises Bancárias: Américas, Europa.....................123

Tabela 4.1 - Objetivos da Regulação............................................................130

Tabela 4.2- Dez maiores transações de resolução de insolvência....................149

Tabela 5.1- O Custo de Resgatar Bancos em Diversos Países...........................197

Tabela 5.2 - Utilização da disponibilidade de liquidez no SFN........................198

Tabela 6.1- Pontuação de eficiência por país...................................................212

Tabela 6.2 - Subsídio de TBTF como percentual dos lucros...........................217

Tabela 6.3 - Fundos de Renda Fixa..............................................................234

Tabela 7.1 - Estatísticas Descritivas..........................................................259

Tabela 7.2 - Probabilidade de Crise Sistêmica e Concorrência........................260

Tabela 7.3 - Testes de Robustez para a Probabilidade de Crise.......................264

Tabela 7.4 - Probabilidade de Crise por Regiões.............................................267

Tabela 7.5 - Probabilidade de Crise Por Regiões - Robustez............................268

Tabela 7.6 - Crise Sistêmica e Estrutura da Concorrência..............................275

Tabela 7.7 - Concorrência e Estrutura Regulatória.......................................277

Tabela 7.8 - Concorrência e Estrutura Regulatória - Critérios de Voigt.........279

Tabela 7.9 - Estrutura da Concorrência e Competição - Robustez.................281

Tabela 8.1- Maiores Bancos por Capitalização de Mercado............................289 


\section{Gráficos}

Gráfico 3.1 - Seguro Depósito e Exposição ao Risco.............................113

Gráfico 5.1 - Retorno Sobre Ativos..................................................178

Gráfico 5.2 - Custos Operacionais.......................................................179

Gráfico 6.1 - Escala Eficiente e Bancos Brasileiros..............................211

Gráfico 6.2 - Ativos vs. FGC...............................................................220 


\section{Parte I}

\section{Introdução}




\section{Capítulo 1}

\section{Introdução}

“(...) setores existem (...) em que, por diversas razões de ordem pública, a entrada e permanência são controladas. (...) Essa regulamentação visa a garantir a higidez $e$ segurança do mercado. (...) O problema é que essa garantia de segurança cria normalmente, por si só, condições propícias à formação de posições dominantes.

(...)

Pode-se dizer que os agentes seriam nesses mercados estruturalmente incentivados a práticas anticoncorrenciais, pois a existência natural de barreiras à entrada, somada às condições instáveis da demanda, faz com que esses mercados sejam particularmente suscetíveis a práticas anticoncorrenciais." ${ }^{1}$

A relação entre o direito concorrencial e a regulação da atividade econômica tem sido objeto de intensas discussões nas mais diferentes áreas. Setores como a telefonia, a energia elétrica, o saneamento básico, para enumerar apenas alguns, foram objeto de exame da compatibilidade entre o regime da concorrência e a regulação.

É na regulação financeira, entretanto, que o debate se torna mais acirrado. Seja pela crença em uma maior independência das agências da área financeira e de mercado de capitais ${ }^{2}$, seja por conta dos graves efeitos advindos

${ }^{1}$ C. SAlomão Filho, Regulação da Atividade Econômica: Princípios e Fundamentos Jurídicos, São Paulo: Malheiros, 2001, passim, pp. 46-49.

2 Cf. I. C. RibeIro, Ivan C.; Cost Benefit Analysis in Financial Regulation: First Lessons from the 2008 Crisis, Revista DE Direito Bancário E do Mercado De CAPITAis, Ano 13, Vol. 50, Out- 
dos momentos de pânico financeiro3 ${ }^{3}$ é nesse setor que se argumenta pela necessidade e existência de verdadeira imunidade concorrencial, ou ao menos de controles mitigados da concorrência.

Nos momentos de instabilidade, em especial, observamos o manejo de um grande aparato de instrumentos e métodos para lidar com o sistema financeiro e seus agentes, evitando resultados indesejados. Nem sempre nesses instantes estaremos falando de uma regulação em senso estrito. Se observássemos a discussão feita pela OCDE - Organização para a Cooperação e Desenvolvimento Econômico (detalhada mais adiante, na seção 4.1.2 do capítulo 4), concluiríamos que o governo alcança os objetivos traçados para o sistema financeiro utilizando-se de instrumentos como a supervisão, a dissuasão moral associada a instrumentos de mercado, o oferecimento de garantias e seguros, o oferecimento de facilidades de crédito e liquidez, programas de subsídios e crédito direcionado, a propriedade governamental pura e simples de alguns participantes do mercado e, por fim, a regulação em senso estrito ${ }^{4}$.

Neste trabalho se examina a compatibilidade do regime concorrencial com essas possibilidades de intervenção no domínio econômico. Analisa-se tanto a regulação propriamente dita como a intervenção direta, esta última típica de momentos de pânico financeiro como o da Crise Financeira de 2008, além de outros instrumentos.

A hipótese teórica e empírica é a de que não apenas existe essa compatibilidade, mas a de que existe uma dupla implicação nessas relações. Medidas regulatórias e de intervenção têm impactos sobre a concorrência,

Dec 2010, pp. 47-75, especialmente nota 31 e texto acompanhando.

3 Diferenciam-se na literatura as corridas bancárias, que ocorrem sempre em relação a uma única instituição financeira, dos pânicos financeiros, que atingem diversas instituições. Veja X. FreIXAS, Xavier; J. C. RocheT, Microeconomics of Banking, MIT Press Books, MIT Press, 2a Edição, 2008, p.21 e nota 3.

4 Segundo a OCDE, "[g]overnments should identify the range of policy instruments and methods at their disposal that are capable of affecting the operation of the financial system, the conduct of its participants, or its outcomes. [b]riefly stated, a policy instrument is a tool used by government to achieve its goals, and thus can be broadly conceived." V. OCDE, Policy 
impactos estes capazes de comprometer a própria eficácia dessas medidas. $\mathrm{O}$ processo de retroalimentação, formando uma relação dialética da regulação, ocorre por conta dos incentivos para que os atingidos pelas modalidades de intervenção no domínio econômico adotem comportamentos estratégicos, acumulando poder no mercado5.

Este trabalho procura elucidar, do ponto de vista teórico, empírico e jurimétrico, os diversos mecanismos resultantes dessa retroalimentação. Após rever os fundamentos da intermediação financeira, da atividade bancária e da regulação, o trabalho propõe uma classificação dialética da regulação financeira. Delineia em seguida três categorias de comportamentos estratégicos, merecedores da atenção do Direito Concorrencial, para que fique resguardada a segurança e a higidez dos mercados financeiros e, principalmente, os objetivos da ordem concorrencial.

\subsection{A Crise de 2007/2008}

A Crise de 2008 e, mais recentemente, a Crise Fiscal na Europa são de grande valia na análise da relação entre a regulação financeira e o Direito Concorrencial. O risco de contágio e as terríveis consequências da quebra de instituições financeiras serviram de justificativa para muitos dos pedidos de desconsideração dos aspectos concorrenciais, e reforçariam mesmo o argumento de que concorrência e estabilidade estão em campos antagônicos.

Assim, a importância da discussão sobre a interação entre o Direito Concorrencial e as possibilidades de intervenção no domínio econômico é bem ilustrada pelos episódios de socorro financeiro durante a Crise de 2008. Os fatos em cada um desses episódios ajudam na compreensão da discussão que será travada neste trabalho, e muitas das condutas anticompetitivas e dos remédios aplicados serão retomados mais adiante nas discussões teóricas. Entre eles, o caso mais emblemático é o da fusão que resultou no Lloyds

Framework for Effective and Efficient Financial Regulation, Paris, 2010.

5 Cf. Salomão Filho, Calixto, Regulação da Atividade Econômica cit. (nota 1 supra), p. 49. 
Banking Group (LBG), mas outros casos de igual importância foram trazidos à Comissão Europeia ou às autoridades antitruste dos Estados Unidos, como os do Royal Bank of Scotland na Escócia, do Commerzbank alemão, do ING holandês e do americano PNC.

\subsubsection{O Caso HBOS}

Em 18 de setembro de 2008 foi anunciada na Inglaterra a compra do HBOS$^{6}$ pelo Lloyds TSB7, com a operação sendo declarada no mesmo dia como de interesse público, para a garantia da estabilidade do sistema financeiro ${ }^{8}$. A lei de concorrência passou a considerar a estabilidade financeira como de interesse público em 24 de outubro, o que autorizaria a realização de fusões e aquisições em violação da regulação das leis antitruste. Essa modificação da legislação concorrencial foi feita quase três semanas depois da aprovação de um pacote de suporte sistêmico aos bancos, em 8 de outubro ${ }^{9}$, resultando em uma injeção de capital na operação de fusão de 17 bilhões de libras ${ }^{10}$.

${ }^{6}$ Embora oficialmente HBOS não seja abreviação de qualquer nome, presume-se que signifique Halifax Bank of Scotland. De fato, a instituição surgiu da fusão do Halifax plc e o Governor and Company of the Bank of Scotland em 2001, conforme L. BACHELOR, HBOS: the issue explained, The Guardian, 04.05.2001, disponível [on-line] in <http://www.guardian.co.uk/money/2001/may/o4/personalfinancenews2> [28.09.2009]. O objetivo da fusão foi anunciado como o de criar um banco que competisse com os assim chamados Big Four, os quatro maiores bancos de varejo do Reino Unido.

7 Terceiro maior banco do Reino Unido - os assim chamados Big Four eram à época, por ordem de tamanho, o HSBC Bank, o Royal Bank of Scotland Group, o Lloyds Banking Group e o Barclays.

${ }^{8} \mathrm{O}$ anúncio foi feito pelo Secretário de Estado para Negócios, Empresas e Reforma da Regulação.

${ }^{9}$ A discussão dos principais fatos do caso pode ser encontrada em J. VICKERS, The financial crisis and competition policy: some economics, GCP - The Online Magazine for Global Competition Policy, 2008, disponível [on-line] in <http://www.ucl.ac.uk/laws/jevons/docs/Vickers_Dec_o8.pdf>, [01/10/2009] e também em H. DAVIES QC e R. BLAKELEY, Merger Action Group v Secretary of State for Business, Enterprise and Regulatory Reform, GCP - The Online Magazine for Global Competition Policy, 2009, disponível [on-line] in <http://www.globalcompetitionpolicy.org/index.php? $\mathrm{id}=1693$ \&action $=907>$, [01/10/2009].

10 O Lloyds TSB condicionou a realização da oferta de aquisição do HBOS em sérias dificuldades por conta da excessiva alavancagem, e as práticas de negócio arriscadas, à capitalização pelo governo. A capitalização foi completada em 19 de janeiro de $2009 \mathrm{com}$ a aquisição de uma participação pelo governo de $43,5 \%$ no banco resultante (13 bilhões de libras em ações 


\subsection{A Evolução da Regulação Financeira}

Ao examinar a relação da substituição da regulação tradicional por uma que aumente a competição, deve-se considerar que a indústria financeira na maior parte do mundo moveu-se para uma nova fase. Nessa etapa, a concorrência em setores tradicionais da atividade bancária, promovida tanto por membros da indústria como por instituições não financeiras, tem forçado a especialização e a inovação. As margens têm decrescido nos países que conseguiram aperfeiçoar sua indústria financeira através da concorrência, bancos têm procurado receitas em outros mercados (internacionalização) e através do desenvolvimento de novos produtos, entre outros desdobramentos.

O resultado é que os sistemas bancários mais modernos, aptos a financiar o desenvolvimento e a inovação, são especializados, com estruturas enxutas, e operando através de diversos parceiros no mercado. Mesmo as atividades de empréstimo, tradicionalmente entendidas como uma tarefa não divisível e típica de bancos comerciais, passaram por essa modernização. Agentes se especializaram no trabalho de originar, na securitização, financiamento e gestão do risco em mercados tão tradicionais quanto o de cartões de crédito e o de financiamento do consumo. Não é o caso do Brasil e outros países em desenvolvimento, nos quais se opera a concentração e se observa o gigantismo de umas poucas instituições.

Assim, propomos neste estudo o que seria essa nova fase da indústria, e apontamos através de um exame empírico as razões para classificar o Brasil em uma fase ainda anterior desse desenvolvimento. 


\subsection{Hipóteses e Desenvolvimento do Trabalho}

A tese neste estudo é a de que tanto as medidas de contenção de crise quanto a regulação financeira em tempos de normalidade econômica têm duas características em comum. Em primeiro lugar, ambas têm impactos diretos sobre a concorrência. Em segundo lugar, elas fornecem incentivos para que os agentes atingidos procurem, através de um comportamento oportunista, aumentar seu poder no mercado e tomar riscos excessivos.

Mesmo nesta pequena introdução, pode-se observar esses efeitos. Recapitalizações feitas com dinheiro público são anunciadas pelos bancos como vantagens competitivas, a exemplo do caso do $\mathrm{ING}^{56}$, no intuito de tomar fatias de mercado dos concorrentes. Os esquemas de garantia de ativos e de liquidez são utilizados para oferecer preços baixos em condições que caracterizam concorrência desleal, e o socorro de bancos problemáticos acaba por premiar aqueles que têm modelos de negócio insustentáveis ou que assumiram riscos excessivos.

A regulação financeira também apresenta efeitos semelhantes. As exigências de capital mínimo podem funcionar como barreiras à entrada, para um primeiro exemplo. A concorrência em determinadas áreas de negócio por instituições não financeiras, que tem proporcionado um alento de concorrência e inovação em diversas partes do mundo, pode não funcionar em um ambiente regulatório muito restritivo. De fato, no caso da regulação financeira, o que tem se chamado de desregulação é, na realidade, a substituição de regulações que distorcem em excesso a concorrência por regulamentos mais específicos e causadores de menos distorções, de caráter prudencial e sistêmico.

A característica comum entre a regulação financeira ordinária e as medidas de contenção de crises é, assim, a sua relação íntima com o regime da concorrência. Não existe nenhuma espécie de incompatibilidade, mas uma

${ }^{56}$ Conforme nota 28 retro e texto acompanhando. 
relação de dupla causalidade que exige um grande escrutínio dos aspectos concorrenciais nos dois casos. Como se vê no regime da União Europeia e dos Estados Unidos, decisões destinadas a aplacar o pânico financeiro e a reduzir a chance de contágio entre instituições financeiras devem vir acompanhadas de medidas para reduzir seus efeitos anticompetitivos. Da mesma forma, medidas regulatórias para tempos normais têm aspectos concorrenciais não negligenciáveis, e a modernização da indústria financeira exige constante atenção aos impactos sobre o poder no mercado dos agentes.

Este trabalho propõe, para realizar o exame dos incentivos trazidos pela regulação, uma classificação dialética. O sistema bancário, baseado no sistema de reservas fracionário, no contrato de depósito e na característica de empréstimo do depositante ao banco desse contrato, convive com falhas fundamentais em seu modelo. Esses aspectos serão explorados em profundidade nos próximos capítulos, mas entre suas consequências mais visíveis estão o pânico financeiro, a tomada excessiva de risco e o chamado risco moral. Na classificação aqui proposta, as regulações primárias lidam com essas falhas fundamentais, gerando, entretanto, outros incentivos e novas falhas. Normas secundárias lidam com esses novos problemas, e nesse instante as instituições financeiras começam a se valer de comportamentos estratégicos para contornar essas regulações e acumular poder no mercado. As normas que lidam com esses comportamentos estratégicos, familiares à literatura sobre regulação, são as regulações terciárias.

Outro tipo de comportamento estratégico, não considerado tradicionalmente na regulação financeira, é aquele que leva em consideração os comportamentos esperados do regulador, em vez do comportamento dos competidores. Esse comportamento leva as instituições financeiras a perseguir o gigantismo, muito além do crescimento capaz de proporcionar algum ganho de escala, escopo ou eficiência custo. Este trabalho propõe então a regulação quaternária, inteiramente fundada nos remédios do Direito Concorrencial e capaz de apresentar resposta a esses comportamentos estratégicos. Serão 
desenvolvidos e apresentados três grupos de comportamentos estratégicos, o Aumento Não Eficiente de Participação no Mercado, a Saturação Anticompetitiva de Mercados e o Bloqueio da Modernização PróCompetitiva.

Os modelos aqui desenvolvidos e discutidos partem da constatação de que esses agentes procuram aumentar o seu poder no mercado valendo-se de incentivos propiciados pela própria regulação. A Crise de 2008 tornou claro, como fica demonstrado no presente estudo, que as instituições financeiras valem-se da garantia implícita de resgate pelo governo (o too big to fail), não apenas para ganhar mercado à custa dos concorrentes, mas também para assumir comportamentos de risco. A regulação e a atuação do governo em momentos de crise, em consequência, acabam funcionando no sentido de aumentar o risco sistêmico, e não de contê-lo.

Esses agentes também se valem, como se verá em nossa discussão, do aumento do número de agências e do seguro bancário como ferramentas para aumentar seu poder no mercado e para assumir riscos intoleráveis. Por fim, há que se considerar a reação dos bancos e instituições financeiras tradicionais, que resistem à modernização como forma de evitar a redução de seu poder no mercado.

Para desenvolver essas hipóteses, este trabalho está organizado em oito capítulos, incluindo esta introdução. O capítulo 2 desenvolve o conceito de falhas essenciais da intermediação financeira, apresentando também os conceitos fundamentais da teoria de intermediação, além dos modelos competição monopolística da seção 2.2.4. Esses modelos são particularmente importantes para a fundamentação do comportamento estratégico classificado como Saturação Anticompetitiva de Mercados, trazendo discussões que inovam em relação à literatura tradicional.

O capítulo 3 discute as falhas e os incentivos proporcionados pela regulação bancária em sua forma tradicional. Lida em sua maior parte com as fragilidades do contrato de depósito e as soluções para que se evitem as 
corridas bancárias. A seção 3.3.5 apresenta importante contraponto à chamada Teoria do Valor de Franquia, que visa justificar o acúmulo de poder no mercado e seu exercício pelos bancos como uma forma de gerar rendas e, assim, aumentar o valor de franquia, reduzir a exposição a riscos e diminuir as chances de corrida bancária.

O capítulo 4 apresenta a classificação dialética sugerida neste trabalho, mostrando também as regulações em espécie e os incentivos proporcionados aos agentes por essas regulações.

O capítulo 5 discute a desregulação e a modernização da indústria, mostrando por que o modelo de megabancos é ultrapassado e perigoso. O capítulo discute ainda a competição e a estabilidade bancária.

O capítulo 6 apresenta e discute em profundidade os comportamentos estratégicos sugeridos como objeto da regulação quaternária. São discutidas também as evidências quanto às economias de escala, de escopo e do aumento da eficiência custo.

O capítulo 7 desenvolve um modelo de inferência jurimétrica para a análise da compatibilidade entre a concorrência e a estabilidade bancária.

O capítulo 8 conclui mostrando o prejuízo do modelo de megabancos ao desenvolvimento econômico e à inovação, e apresenta sugestões de políticas públicas. 


\section{REFERÊNCIAS BIBLIOGRÁFICAS}

ACEMoglu, D.; Johnson, S.; RoBInson, J.; The colonial origins of comparative development: An empirical investigation, American Economic Review, v. 91, p. 1.369-1.401, 2001.

Aghion, Philippe; Bolton, Patrick; An incomplete contracts' approach to financial contracting, Review of Economic Studies, 59 (3), 1992, pp. 473-494.

AKERLOF, George A.; The Market for 'Lemons': Quality Uncertainty and the Market Mechanism, Quarterly Journal of Economics, 1970, vol. 84(3), pp. 488-500.

ALlison, Paul D., WATERMAN, Richard, Fixed-effects negative binomial regression models, Sociological Methodology 32, 2002.

AMARAL, Pedro S., QUINTIN, Erwan, Making finance matter in FRBD Working Paper Series 0104 (2004).

AMERICAN BAR ASSOCIATION, Bank mergers and acquisitions handbook, Chicago, Section of Antitrust Law, ABA, 2006.

Section on Antitrust Law, Monograph 24, "Federal Statutory Exemptions from Antitrust Law", 2007.

ANTUNES, António; CAVAlCANTI, Tiago; Villamil, Anne, Intermediation costs, investor protection and economic development, in II Encontro de Políticas Públicas e Crescimento Econômico, Fortaleza, CAEN/EPGE, maio de 2005.

AREEDA, Phillip, HovenKAMP, Herbert, Fundamentals of antitrust law, $3^{\mathrm{a}}$ Ed, New York, Aspen Law \& Business, 2002.

ARrow, K.; The Role of Securities in the Optimal Allocation of Risk-bearing, Review of Economic Studies, Vol. 31(2), 1964, pp. 91-96.

DeBreu, G.; Existence of an Equilibrium for a Competitive Economy, Econometrica, Vol. 22(3), 1954, pp. 265-290.

ASCARELLI, Túlio, Teoría de la concurrencia y de los bienes inmateriales, in Revista Crítica de Derecho Inmobiliario 485 (1971).

Teoria geral dos títulos de crédito, $2^{\mathrm{a}}$ Ed, São Paulo, Saraiva, 1969. 
Corso de diritto commerciale: introduziona e teoria dell'impresa, $3^{\mathrm{a}}$ Ed, Milão, Giuffrè, 1962, tradução de Comparato, Fábio Konder, in RDM 103 (1996), pp. 87-100.

Problemas das sociedades anônimas e direito comparado, $2^{\mathrm{a}} \mathrm{Ed}$, São Paulo, Saraiva, 1969.

AUSUBEL, Lawrence M., The failure of competition in the credit card market, in American Economic Review 81:1 (1991), pp. 50-81.

BAER, William, A Report on Recent Antitrust Developments at the FTC, comentários feitos na ABA Section of Antitrust Law em 9 de agosto de 1995, consultado em 17 de maio de 2011 em http://www.ftc.gov/speeches/other/baersp.shtm.

BAGEHOT, Walter; Lombard Street A Description of the Money Market, 1873, reimpressão de CreateSpace, New York, 2011, 15op.

BAILEY, Norman T. J.; The mathematical theory of infectious diseases and its applications; 2a edição, Londres: Griffin, 1975, 413 p.

BAIN, Joe S.; Relation of profit rate to industry concentration: American manufacturing, 1936-1940; Quarterly Journal of Economics, 65(3), 1951, pp. 293-324.

BAKER, Dean; MCARThur, Travis; The Value of the 'Too Big to Fail' Big Bank Subsidy, novembro de 2009, Center for Economic and Policy Research (CEPR).

BALTENSPERGER, Ernst; Alternative Approaches to the Theory of the Banking Firm, Journal of Monetary Economics, Volume 6(1), 1980, pp. 1-37.

Banco Central do Brasil, Diretoria de Política Econômica, Funções do Banco Central, janeiro de 2012.

BANCO Mundial, Finance for Growth: Policy Choices in a Volatile World, Oxford: Oxford University Press e Banco Mundial, 2001.

BANERJEE, Abhijit V.; A Simple Model of Herd Behavior, Quarterly Journal of Economics, Vol. 107, no 3, 1992, pp. 797-817.

BARRO, Robert. Economic Growth in a Cross Section of Countries, Quarterly Journal of Economics, 106:2 (1991), pp. 407-443.

BARRoso, Luís Roberto, A ordem econômica constitucional e os limites à atuação estatal no controle de preços, in RDJ 14 (2002).

BARTH, James R.; Trimbath, Susanne; YAGO, Glenn; The Savings and Loan Crisis: Lessons from a Regulatory Failure, Santa Monica, CA: Milken Institute, 2004, 392p.

BAUMOL, William J., PANZAR, John C., Willig, Robert D., Contestable markets and the theory of industry structure, San Diego, Harcourt Brace Jovanovich, 1982. 
BEck, Thorsten, DemirgüÇ-Kunt, Asli, Levine, Ross, Bank concentration, competition, and crises: first results, in Journal of Banking \& Finance 30 (2006), pp. 1582-1603.

BECKER, Gary. A theory of competition among pressure groups for political influence. Quarterly Journal of Economics, Cambridge, Mass., v.98 n.3, agosto de 1983.

BELAISCH , Agnes; Do Brazilian Banks Compete? , IMF working paper 03-113, 2003.

Bencivenga, Valerie R., Smith, Bruce D., Financial intermediation and endogenous growth, in Review of Economic Studies 58:2 (1991), pp. 195-209.

Benston, George J., Market Discipline: The Role of Uninsured Depositors and Other Market Participants, in R. Randall (editor), Safeguarding the Banking System in an Environment of Financial Cycles, Conference Series 7, Boston: Federal Reserve Bank of Boston, 1993, pp. 65-95.

; Interest Payments on Demand Deposits and Bank Investment Behavior, Journal of Political Economy, 72, outubro de 1964, pp. 431-449.

; SMITH, Clifford W. Jr.; A Transactions Cost Approach to the Theory of Financial Intermediation, Journal of Finance , Vol. 31, No. 2, Papers and Proceedings of the Thirty-Fourth Annual Meeting of the American Finance Association Dallas, Texas December 28-30, 1975 (May, 1976), pp. 215231.

BERCOVICI, Gilberto, A Constituição e o papel do Estado no domínio econômico, in RABDC 2 (2002), pp. 117-129.

A ordem econômica no espaço, In Cardoso Jr, José Celso, Castro, Paulo R. Furtado de, Motta, Diana Meirelles da (Orgs), A Constituição brasileira de 1988 revisitada: recuperação histórica e desafios atuais das políticas públicas nas áreas regional, urbana e ambiental, Brasília, Instituto de Pesquisa Econômica Aplicada, 2009, pp. 183-201.

Constituição econômica e desenvolvimento, in RABDC 5 (2004), pp. 203-219.

Constituição econômica e desenvolvimento: uma leitura a partir da Constituição de 1988, São Paulo, Malheiros, 2005, 190 p.

, O estado de exceção econômico, in RABDC 7 (2005), pp. 193-199.

MASSONETTO, L. F., Limites da regulação: esboço para uma crítica metodológica, in RDPE 25 (2009), pp. 137-147.

BERG, Sigbjørn A.; KIM, Moshe; Banks as Multioutput Oligopolies: An Empirical Evaluation of the Retail and Corporate Banking Markets, Journal of Money, Credit and Banking, Vol. 30, No. 2, 1998, pp. 135-153. 
BERGER, Allen N.; The Profit-Structure Relationship in Banking: Tests of Market Power and Efficiency-Structure Hypothesis, Journal of Money, Credit and Banking, vol. 27, 1995, pp. 404-431.

DemirgüÇ-Kunt, Asli, Levine, Ross, Haubrich, Joseph G., Introduction - bank concentration and competition: an evolution in the making, in FRBC Proceedings, 2004, pp. 433-451.

; DICK, Astrid A.; Entry into banking markets and the early-mover advantage, Journal of Money, Credit and Banking, volume 39(4), 2007, pp. $775-807$.

; HANNAN, Timothy H.; The price-concentration relationship in banking, Review of Economics and Statistics 71:2 (1989), pp. 291-299.

; HUMPHREY, David B.; Megamergers in Banking and the Use of Cost Efficiency as an Antitrust Defense; Antitrust Bulletin, Vol. 37, 1992, pp. 541-600.

; The dominance of inefficiencies over scale and product mix economies in banking, Journal of Monetary Economics, Volume 28(1), 1991, pp. 117-148.

; KASHYAP, Anil K.; SCALISE, Joseph M.; GERTLER, Mark; FRIEDMAN, Benjamin M.; The Transformation of the U.S. Banking Industry: What a Long, Strange Trip It's Been; Brookings Papers on Economic Activity, Vol. 1995, No. 2, 1995, pp. 55-218.

; MESTER, Loretta J.; Inside the black box: What explains differences in the efficiencies of financial institutions? Journal of Banking \& Finance, Volume 21(7), 1997, pp. 895-947.

; UDELL, Gregory F.; A more complete conceptual framewortk for SME finance, Journal of Banking and Finance, Vol. 30(11), 2006, pp. 29452966.

; Small business credit availability and relationship lending: the importance of bank organizational structure, Economic Journal 112:477, 2002, pp. F32-F53.

; Some Evidence on the Empirical Significance of Credit Rationing, Journal of Political Economy, Vol. 100(5), 1992, pp. 10471077.

BERNANKE, Ben S., Credit in the macroeconomy, in FRBNY Quarterly Review Spring 1992-1993, pp. 50-70.

, BLINDER, Alan S., Credit, money, and aggregate demand, in American Economic Review 9:4 (1988), pp. 435-439.

GERTLER, Mark, Inside the black box: the credit channel of monetary policy transmission, in Journal of Economic Perspectives 9:4 (1995), pp. 27-48. 
, Lown, Cara S., The credit crunch, in Brookings Papers on Economic Activity, 2 (1991), pp. 205-247.

BernsteIn, Marver H. ; Regulating Business by Independent Commission, 1955, reimpressão de 1977, Connecticut: Greenwood Press, 306p.

BESANKo, David A., THAKOR, Anjan V., Banking deregulation: allocational consequences of relaxing entry barriers, Journal of Banking \& Financing 16 (1992), pp. 909-932.

BHARgava, Rahul, Fraser, Donald R., On the wealth and risk effects of commercial bank expansion into securities underwriting: An analysis of Section $2 O$ subsidiaries, Journal of Banking \& Financing, 22, 1998, pp. 447465.

BISIGNANO, Joe; Structure of Financial Intermediation, Corporate Finance and Central Banking, Bank for International Settlements, mimeo, 1990.

BLACK, Fischer; Scholes, Myron; The Pricing of Options and Corporate Liabilities, Journal of Political Economy, Vol. 81, no. 3, 1973, pp. 637-654.

BLISS, Richard T.; ROSEN, Richard J.; CEO compensation and bank mergers, Journal of Financial Economics, 2001, volume 61(1), pp. 107-138.

Blume, Marshall; Allen, Franklin; Does Short-selling Need the SEC's Oversight? Entrevista para o Knowledge@Wharton, julho de 2008, publicado em http://knowledge. wharton.upenn.edu/article.cfm? articleid=2021, consultado em novembro de 2010.

BOHÁCEK, Radim, MENDIZÁBAL, Hugo Rodriguez, Credit markets and the propagation of monetary policy shocks, in Journal of Money, Credit and Banking 39:6 (2006), pp. 1429-1455.

Bond, Eric W.; CROCKER, Keith J.; Bank Capitalization, Deposit Insurance, and Risk Categorization, Journal of Risk and Insurance, Vol. 6o, No. 4, 1993, pp. 547-569.

Boone, Jan; A New Way to Measure Competition, The Economic Journal, Volume 118, Issue 531, pp. 1245-1261, 2008.

; VAN OURS, Jan C.; VAN DER WIEL, Henry; How (Not) to Measure Competition, TILEC Discussion Paper No. 2007-014, 2007.

BovEnZI, John F., Muldoon, Maureen F.; Failure-resolution methods and policy considerations, FDIC Banking Review, Vol. 3, 1990, pp. 1-11.

Box, G. E., JENkIns, G. M, Time series analysis: forecasting and control, San Francisco, Holden Day, 1976.

BoYD, John H., DE NiCOLÒ, Gianni, Jalal, Abu M., Bank competition, risk and asset allocations, in IMF Working Paper Series 143 (2009).

; The Theory of Bank Risk Taking and Competition

Revisited, The Journal of Finance, Volume 6o(3), 2005, pp. 1329-1343. 
; GERTLER, Mark; Are Banks Dead? Or Are the Reports Greatly Exaggerated? Federal Reserve Bank of Minneapolis Quarterly Review, Vol. 18(3), 1994.

BRESNAHAN, Timothy F., The oligopoly solution is identified, in Economic Letters 10 (1982), pp. 87-92.

; Empirical Studies of Industries with Market Power, in Richard Schmalensee e Richard Willig, Handbook of Industrial Organization, Volume 2, Capítulo 17, 1989, pp. 1011-1057.

BRIONES, Ignacio, VILLELA, André, European banks and their impact on the banking industry in Chile and Brazil: 1862-1913, in Oesterreichische Nationalbank Working Paper 108 (2005).

BROECKER, Thorsten; Credit Worthiness Tests and Interbank Competition, Econometrica, 58(2), 1990, pp. 429-452.

BROWNE, Frank; Efficiency and Financial Fragility in Irish Banking: Causes, Consequences and Policy Recommendations, Bank of Ireland Research Paper no. 4/R/92, 1992.

BrunNer, Karl, Meltzer, Allan H., Money and credit in the monetary transmission process, in American Economic Review, 78:2 (1988), pp. 446451.

BRYANT, John; A model of reserves, bank runs, and deposit insurance, Journal of Banking \& Finance, 4(4), 1980, pp. 335-344.

Bulgarelli, Waldírio, Títulos de crédito, São Paulo, Atlas, $17^{\mathrm{a}} \mathrm{Ed}, 2001$.

Calavita, Kitty; Pontell, Henry N.; Tillman, Robert H.; Big Money Crime: Fraud and Politics in the Savings and Loan Crisis, Berkeley e Los Angeles: University of California Press, Paperback Edition, 1999, 264p.

CAlomiris, Charles W., Financial Innovation, Regulation, and Reform, Cato Journal, vol. 29, 2009, pp. 65-91.

University Press, 2000.

U.S. Bank Deregulation in Historical Perspective, Cambridge

Building an Incentive-compatible Safety Net, Journal of Banking \& Finance, vol. 23(10), 1999, pp. 1499-1519.

, GORTON, Gary B., The origins of banking panics: models, facts, and bank regulation, in Hubbard, Glenn, Financial markets and financial crises, Chicago, University of Chicago, 1991.

; WhITE, Eugene N.; The Origins of Federal Deposit Insurance, in Charles W. Calomiris, U.S. Bank Deregulation in Historical Perspective, Cambridge University Press, 2000, pp. 164-211, especialmente pp. 165-166.

CAlvo, Guillermo A.; On the Time Consistency of Optimal Policy in a Monetary Economy, Econometrica, Vol. 46(6), 1978, pp. 1411-1428. 
CAmeron, A. Colin, Triverdi, Pravin K., Regression analysis of count data, Cambridge, Cambridge University Press, 1998.

CARlton, Dennis W, Perloff, Jeffrey M., Modern industrial organization, $4^{\text {a }}$ Ed, Reading, Addison-Wesley Longman, 2005. pp. $637-658$.

The rigidity of prices, in American Economic Review 76:4 (1986),

CARTER, David A.; MCNulty, James E.; Verbrugge, James A.; Do Small Banks have an Advantage in Lending? An Examination of Risk-Adjusted Yields on Business Loans at Large and Small Banks, Journal of Financial Services Research, Volume 25, Numbers 2-3 (2004), 233-252.

Chan, Yuk-Shee; Greenbaum, Stuart I.; Thakor, Anjan V.; Is Fairly Priced Deposit Insurance Possible?, Journal of Finance, vol. 47(1), 1992, pp. 227-245.

CHARI, Varadarajan V.; JAGANnATHAN, Ravi; Banking Panics, Information, and Rational Expectations Equilibrium, Journal of Finance, Vol. 43(3), 1988, pp. 749-761.

CIOCCA P. Supervision: one or more institutions?, in BIS Review 39 (2001).

ClaEsSEns, Stijn, LAEVEn, Luc, What drives bank competition? Some international evidence, Journal of Money, Credit and Banking 36:3, 2004, pp. 563-583.

COASE, Ronald Harry, The nature of the firm, in Economica 4:16 (1937), pp. 386-4052.

COCCORESE, Paolo, Market power in local banking monopolies, in Journal of Banking \& Finance 33 (2009), pp. 1196-1210.

COMITÊ DE SUPERVISÃO E REFORMA DO GOVERNO DA CÂMARA DOS REPRESENTANTES, The Financial Crisis and the Role of Federal Regulators, Audiência Pública, 23 de outubro, 2008.

Comparato, Fábio Konder, SAlomão Filho, Calixto, O poder de controle na sociedade anônima, $4^{\mathrm{a}}$ Ed, São Paulo, Forense, 2005.

, O Ministério Público na defesa dos direitos econômicos, sociais e culturais, in RFDUFMG 40 (2002), pp. 67-50.

, The economic order in the Brazilian Constitution of 1988, in The American Journal of Comparative Law, 38 (1990), pp. 753-771.

Na proto-historia das empresas multinacionais - o Banco Medici de Florenca, in RDM 54 (1984), pp. 105-111. (1983), pp. 95-104.

Notas retificadoras sobre seguro de crédito e fiança, in RDM 51 O repasse bancário de recursos externos, in RDM 76 (1989), pp. 49-53. 
, O seguro de crédito, São Paulo, Revista dos Tribunais, 1968.

CORTS, Kenneth S.; Conduct Parameters and the Measurement of Market Power; Journal of Econometrics, Vol. 88 (2), 1999, pp. 227-250.

DAVIES Q. C., Helen, BlaKelEY, Richard, Merger Action Group $v$ Secretary of State for Business, Enterprise and Regulatory Reform, in GCP - The Online Magazone for Global Competition Policy, release 1 (2009).

DE ChIARA, José Tadeu, Moeda e ordem jurídica, Tese de doutorado, FDUSP, 1986.

DE LEÓN, Ignacio, An institutional assessment of antitrust policy: the Latin American experience, Alphen aan den Rijn, Kluwer Law International, 2009.

DE LUCCA, Newton, Reflexões sobre os 40 anos do Banco Central, in RDBMCA 35 (2007), pp. 194-206.

, A influência do pensamento de Túlio Ascarelli em matéria de títulos de crédito no Brasil, in RTRF3R 69 (2005), pp. 11-39.

A aplicação do Código de Defesa do Consumidor à atividade bancária, in RIADP 2 (2000), pp. 207-214.

, Aspectos da teoria geral dos títulos de crédito, Pioneira, 1979.

158 (1977).

Análise do crédito ao consumidor no direito brasileiro, in RTJE

, Taxa de juros, in CEJ 1 (1976).

DE MELlo, João M. P.; Estrutura, Concentração e Estabilidade, in Márcio Garcia e Fabio Giambiagi, Risco e Regulação, Rio de Janeiro: Elsevier, 2010, pp. 111-123.

; Atos de Concentração e Conduta no Setor Bancário, in Sistema Financeiro - o Desafio da Concorrência, Daniel K. Goldberg (org.), São Paulo: Editora Singular, 2008, pp. 43-110.

DEgRYSE, Hans; KIM, Moshe; ONGENA, Steven; Microeconometrics of Banking: Methods, Applications and Results, New York: Oxford University Press, 2009.

; Ongena, Steven; Competition and Regulation in the Banking Sector: A Review of the Empirical Evidence on the Source of Bank Rents, in Anjan V. Thakor e Arnoud W. A. Boot (Editores), Handbook of Financial Intermediation and Banking, Amsterdam: Elsevier, 2008.

; _ _ Competition and Regulation in the Banking Sector:

A Review of the Empirical Evidence on the Source of Bank Rents, mimeo, 2005a.

; Distance, Lending Relationships, and Competition,

Journal of Finance, vol. 6o(1), 2005b, pp. 231-266. 
DELIS, Manthos D., Tsionas, Efthymios G., The joint estimation of bank-level market power and efficiency, in Journal of Banking \& Finance 33 (2009), pp. 1842-1850.

DemirgüÇ-Kunt, Asli; Detragiache, Enrica, Does Deposit Insurance Increase Banking System Stability? An Empirical Investigation, Journal of Monetary Economics, vol. 49(7), 2002, pp. 1373-1406.

; The determinants of banking crises in developing and developed countries, IMF Staff Papers 45, Washington, International Monetary Fund, 1998, 81-109.

; HuIZINGA, Harry; Do we need big banks? Evidence on performance, strategy and market discipline, World Bank, Policy Research Working Paper 5576, fevereiro de 2011, 45p.

; LAEVEN, Luc; LEVINE, Ross; Regulations, market structure, institutions, and the cost of financial intermediation, in Journal of Money, Credit and Banking 36:3 (2004), pp. 593-622.

DEMSETZ, Harold; Industry structure, market rivalry, and public policy, in Journal of Law and Economic 16:1 (1973), pp. 1-10.

DemSETz, Rebecca S.; Strahan, Philip E.; Diversification, Size, and Risk at Bank Holding Companies, Journal of Money, Credit and Banking, Vol. 29(3), 1997, pp. 300-313.

DEPARTMENT OF JUSTICE, Bank Merger Competitive Review -- Introduction and Overview, 1995, disponível em http://www.justice.gov/atr/public/guidelines/6472.htm, consultado em 22/11/2009.

, Comunicado à imprensa em 11 de dezembro de 2008, disponível em http://www.justice.gov/atr/public/press_releases/2008/240315.pdf e consultado em 03 de maio de 2011.

Detragiache, Enrica; DEMIRGÜÇ-KunT, Asli; Cross-Country Empirical Studies of Systemic Bank Distress: A Survey, IMF Working Papers 05/96, International Monetary Fund, 2005.

Devereux, Michael B., SMith, Gregor W., International risk sharing and economic growth, in International Economic Review 35:4 (1994), pp. 535-550.

DEWATRIPONT , Mathias; TiRole, Jean, The Prudential Regulation of Banks, The Walras-Pareto Lectures, Cambridge, MA: MIT Press, 1994, pp. 31-45.

DeYoung, Robert, HunTER, William C., UdELL, Gregory F., The past, present, and probable future of community banks, in Journal of Financial Services Research 25:2-3 (2004) pp. 85-133.

Diamond, Douglas W.; Financial Intermediation and Delegated Monitoring, Review of Economic Studies, Vol. 51, No. 3, 1984), pp. 393-414. 
; DYBVIG Philip H.; Bank Runs, Deposit Insurance, and Liquidity, Journal of Political Economy, Vol. 91, No. 3, 1983, pp. 401-419.

EATON, B. Curtis; LIPSEY, Richard G.; The Theory of Market Pre-emption: The Persistence of Excess Capacity and Monopoly in Growing Spatial Markets, Economica, Nova Série, Vol. 46, No. 182, 1979, pp. 149-158.

EDWARDS, Franklin R.; Concentration in Banking and Its Effect on Business Loan Rates, Review of Economics and Statistics, Vol. 46(3), 1964, pp. 294300.

EHLERMANN, Claus-Dieter; State Aids under European Community Competition Law, Fordham International Law Journal, Vol. 18, 1994-1995, pp. 410-436.

ENDERS, Walter, Applied econometric time series, ${ }^{\text {a }}$ Ed, New Jersey, John Wiley \& Sons, 2004.

ENNIS, Huberto M., KeISTER, Todd, Bank runs and institutions: the perils of intervention, in American Economic Review 99:4 (2009), pp. 1588-1607.

EREL, Isil; The Effect of Bank Mergers on Loan Prices: Evidence from the U.S., Aug. 2006, artigo apresentado na Corporate Finance of Financial Intermediaries, conferência patrocinada pelo Federal Reserve Bank of New York, pelo Wharton Financial Institutions Center of University of Pennsylvania e pela revista Review of Financial Studies em Setembro de 2006.

EUROPEAN COMPETITION AUTHORITIES - Financial Services Subgroup, Comparative study of competition in retail banking and payments systems markets, Londres, 18 e 19 de abril de 2005 .

FACHAdA, Pedro, FigueIredo, Luiz Fernando, Lundberg, Eduardo, Sistema judicial e mercado de crédito no Brasil, Nota Técnica do Banco Central do Brasil 35 (2003).

FAMA, Eugene F., e FRENCH, Kenneth R., Multifactor explanations of asset pricing anomalies, Journal of Finance, 51(1), março de 1996, pp. 55-84.

FISCHER, Lawrence, JENSEN, Michael, e RoLL, Richard, The adjustment of stock prices to new information, International Economic Review, vol. 10, 1969, pp. 1-21.

FAVA, Vera Lúcia, Modelos de função de transferência e de análise de intervenção, in Vasconcellos, Marco Antonio Sandoval, Alves, Denisard Cnéio de Oliveira (Org.), Manual de econometria: nível intermediário, São Paulo, Atlas, p. 233-243, 2000.

, Alves, Denisard C., Indicador de movimentação econômica, Plano Real e análise de intervenção, in RBE 51:1 (1997), pp. 133-143.

FEDERAL DEPOSIT InSURANCE CORPORATION, FDIC Statement of Policy on Bank Merger Transactions, disponível em 
http://www.fdic.gov/regulations/laws/rules/500o-1200.html, acessado em 22/11/2009.

FEDERAL RESERVE BOARD, Order Approving Formation of a Bank Holding Company and Notice to Engage in Nonbanking Activities, Travelers Group Inc., New York, New York, Citicorp, New York, New York, 23 de setembro de 1998.

Ferguson, Niall, The Ascent of Money: A Financial History of the World, New York: Penguin Books, 2008.

FinANCIAL SERVICES AUTHORITY, A new regulator for the new millennium, janeiro de 2000, acessado em http://www.fsa.gov.uk/pubs/policy/p29.pdf, consulta em 12/11/2011.

FISHER, Irving; The Purchasing Power of Money: Its determination and relation to credit interest and crises, 2a Edição, Nova Iorque: Macmillan, 1992, reimpressão na série Reprints of Economic Classics, Nova Iorque: Augustus M. Kelley, 1963.

FONSECA, Antonio, Limites jurídicos da regulação e defesa da concorrência, Porto Alegre, Sergio Antonio Fabris Editor, 2003.

Forgioni, Paula, A evolução no Direito Comercial Brasileiro: da mercancia ao mercado, São Paulo, Revista dos Tribunais, 2009.

Tribunais, 2008.

Os fundamentos do antitruste, $3^{\text {a }}$ Ed, São Paulo, Revista dos

A interpretação dos negócios empresariais no novo Código Civil brasileiro, in RDM 130 (1995), p. 7-38.

FRANK, Murray Z., MAKSIMOVIC, Vojislav, Trade credit, collateral, and adverse selection, in SSRN Working Paper 87868 (2005).

FREIXAS, Xavier; Rochet, Jean-Charles; Microeconomics of Banking, Cambridge: MIT Press, 2a edição, 2008.

; Fairly priced deposit insurance: Is it possible? Yes.

Is it desirable? No; Finance and banking discussion paper 4, Universitat Pompeu Fabra, Barcelona, 1995.

FRIEDMAN, Benjamin M., Monetary policy without quantity variables, in American Economic Review, 78:2 (1988), pp. 440-445.

Friedman, Milton e SchwarTz, Anna; A Monetary History of the United States, 1867-1960, Princeton: Princeton University Press (NBER), 1963, p. 329.

Fudenberg, Drew; Tirole, Jean, Preemption and Rent Equalization in the Adoption of New Technology, Review of Economic Studies, vol. 52(3), 1985, pp. 383-401. 
$\longrightarrow$ Iorque: Harwood Academic Publishers, 1986.

Gale, Douglas; Hellwig, Martin, Incentive-Compatible Debt Contracts: The One-Period Problem, Review of Economic Studies, vol. 52(4), 1985, pp. 647663.

GALVÃO, A. B., Structural break threshold VARs for predicting US recessions using the spread, in Journal of Applied Econometrics 21 (2006), pp. 463-487.

Garcia, Márcio; Giambiagi, Fabio; Risco e Regulação, Rio de Janeiro: Elsevier, 2010.

GARDNER, William, MULVEY, Edward P., SHAW, Esther C., Regression analyses of counts and rates: Poisson, overdispersed Poisson, and negative binomial models, Psychological Bulletin, 118:3, 1995, pp. 392-404.

GibBons, J. S.; The Banks of New York, Their Dealers, the Clearing House, and the Panic of 1857, Nova Iorque: Greenwood Press, reimpressão da edição de 1859, 1968.

GIBSON, Katherine, The early history and initial impact of NOW accounts, New England Economic Review, Jan/Fev de 1975, pp. 17-26.

GILBERT, R. Alton, Bank market structure and competition: a survey, in Journal of Money, Credit and Banking 16 (1984), pp. 617-645.

Gilbert, Richard J.; Newbery, David M G; Preemptive Patenting and the Persistence of Monopoly, American Economic Review, vol. 72(3), 1982, pp. 514-526.

Goetz, Charles J., McChesney, Fred S., Antitrust law: interpretation and implementation, $4^{\text {a }}$ Ed, New York, Foundation, 2009.

GolDSMITH, Raymond W., 1850-1984: desenvolvimento financeiro sob um século de inflação, Rio de Janeiro, Banco Bamerindus e Harper \& Row, 1986.

Goldstein, Morris; Véron, Nicolas; Too Big to Fail: The Transatlantic Debate, in Jacob Funk Kirkegaard, Nicholas Veron e Guntram B. Wolff (Editores), Transatlantic Economic Challenges in an Era of Growing Multipolarity, Peterson Institute, 2012.

GOODHART, Charles A.; Why do banks need a central bank? Oxford Economic Papers 39 (1), pp. 75-89, 1987.

; Monetary Theory and Practice: The U. K. Experience; London: Palgrave Macmillan, 1983, pp. 23-29.

GORTON, Gary B., Banking panics and business cycles, in Oxford Economic Papers 40 (1988), pp. 751-81.

, Banking theory and free banking history: a review essay, in Journal of Monetary Economics 16:2 (1985), pp. 267-76. 
, HAUBRICH, Joseph, Bank deregulation, credit markets, and the control of capital, in Carnegie-Rochester Conference Series on Public Policy 26 (1987), pp. 189-234.

, HuANG, Lixin; Liquidity, efficiency, and bank bailouts, American Economic Review 94:3 (2004).

, Banking panics and the origin of central banking, in

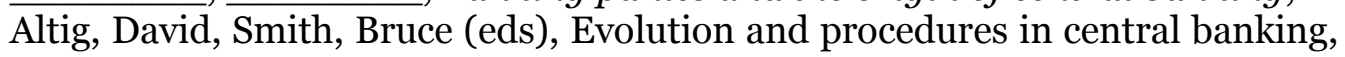
Cambridge, Cambridge University, 2003.

, Mullineaux, Don, The joint production of confidence:

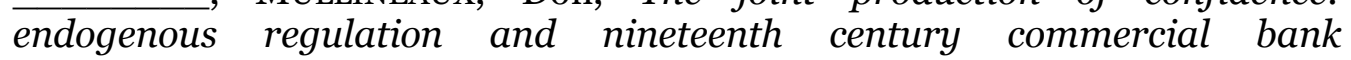
clearinghouses, in Journal of Money, Credit and Banking 19:4 (1987), pp. 45868.

; PennacchI, George G.; Banks and Loan Sales: Marketing Nonmarketable Assets, Journal of Monetary Economics 35 (1995), pp. 389411.

; Rosen, Richard; Corporate Control, Portfolio Choice and the Decline of Banking, Journal of Finance, Vol. 50, 1995, pp. 1377-1420.

SCHMID, Frank, Universal banking and the performance of german firms, in Journal of Financial Economics 58 (2000), pp. 3-28.

WINTON, Andrew, Banking in transition economies: does efficiency require instability? , in Journal of Money, Credit, and Banking 30 (1998), pp. 621-650.

GRAU, Eros Roberto, A ordem econômica na Constituição de 1988, 4ª edição, São Paulo, Malheiros, 1998.

Tribunais, 1981 .

Elementos do direito econômico, São Paulo, Revista dos

Forgioni, Paula Andréa, O Estado, a empresa e o contrato, São Paulo, Malheiros, 2005, $352 \mathrm{p}$.

, CADE vs. BACEN: conflitos de competência entre

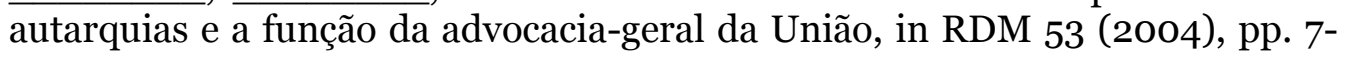
25 .

, Restrição à concorrência, autorização legal e seus limites: Lei n. 8.884, de 1994 e Lei n. 6.729, de 1979 (Lei Ferrari), in Revista de Direito Público, Bélgica, 21 (1998), pp. 107-121.

Greenbaum, Stuart I.; ThaKor, Anjan V.; Contemporary Financial Intermediation, 2a Edição, Amsterdam, Boston, Heidelberg: Elsevier Academic Press, 2007.

GREENE, William, Estimating econometric models with fixed effects, artigo não publicado, 2001, disponível em http://www.stern.nyu.edu/ wgreene, consultado em 23.04.2010. 
Gross, David B., Souleles, Nicholas S., Do liquidity constraints and interest rates matter for consumer behavior? evidence from credit card data, in Quarterly Journal of Economics 117:1 (2002), pp. 149-185.

GROSSMAN, Sanford J., HART, Oliver D., The costs and benefits of ownership: a theory of vertical and lateral integration, in Journal of Political Economy 94:4 (1986), pp. 691-719.

GuIMARÃES, Paulo, The fixed effects negative binomial model revisited, Economic Letters 99 (2008), pp. 63-66.

GURLEY, John G.; SHAW, Edward S.; Money in a Theory of Finance, Washington, DC: Brookings Institute Press, 1960.

HAGEN, Kåre P.; Default Risk, Homemade Leverage, and the ModiglianiMiller Theorem: Note, American Economic Review , Vol. 66, No. 1 (Mar., 1976), pp. 199-203.

Hamilton, James Douglas, Time series analysis, Princeton, Princeton University, 1994.

HANLEY, A. G., Capital markets in the coffee economy: financial institutions and economic change in São Paulo, Brazil, 1840-1905, Tese de Doutorado, Stanford University, 1995.

HANnAN, Timothy H.; BERGER, Allen N.; The Rigidity of Prices: Evidence from the Banking Industry; American Economic Review; Vol. 81, No. 4, 1991, pp. 938-945.

HAUSMAN, Jerry, HALl, Bronwyn H., GRILICHES, Zvi, Econometric models for count data with an application to the patents-R\&D relationship, Econometrica, 52:4, 1984, pp. 909-938.

HAWAWINI, Gabriel, SWARY, Itzhak, Mergers and acquisitions in the U.S. banking industry: evidence from the capital markets, Amsterdam, NorthHolland, 1990.

HAYNES, George W.; OU, Charles; BERNEY, Robert; Small Business Borrowing from Large and Small Banks, in Business Access to Capital and Credit, editado por Jackson L. Blanton, Alicia Williams e Sherrie L. W. Rhine, Conferência de Pesquisa do Federal Reserve System, 1999, pp. 287-327.

HAZEN, Thomas L.; MARKHAM, Jerry W.; A Broker-Dealer Operations Under Securities and Commodities Law, Securities Law Series, Vol. 23, ThomsonWest, 2007.

HELLWIG, Martin, Banking, financial intermediation and corporate finance, in A. Giovanni and C. Mayer (Editores), European Financial Integration, Londres: Cambridge University Press, 1991, pp. 35-63.

HENSEL, Nayantara D.; Strategic management of cost efficiencies in networks: Cross-country evidence on European branch banking, European Financial Management, Volume 9(3), 2003, pp. 333-360. 
Her Majesty's TREasury, Comunicado à Imprensa, consultado em 23/04/2011 em www.hm-treasury.gov.uk/press_23_09.htm.

HETZEL, Robert L.; Too big to fail : origins, consequences, and outlook, Economic Review, Federal Reserve Bank of Richmond, novembro 1991, pp. 315 .

HILBE, Joseph M., Negative binomial regression, Cambridge, Cambridge University Press, 2007.

HIRSHLEIFER, Jack; On the Theory of Optimal Investment Decision, Journal of Political Economy, Vol. 66, No. 4 (Aug., 1958), pp. 329-352.

HIRTLE, Beverly; The impact of network size on bank branch performance, Journal of Banking \& Finance, Volume 31(12), 2007, pp. 3782-3805.

HOLDER, Christopher L., Competitive considerations in bank mergers and acquisitions: economic theory, legal foundations, and the Fed, in FRBA Economic Review, jan-fev, (1993), pp. 23-36.

Holmstrom, Bengt; Tirole, Jean, Financial Intermediation, Loanable Funds, and the Real Sector, Quarterly Journal of Economics, vol. 112(3), 1997, pp. 663-691.

Hong, Harrison; KuBIK, Jeffrey D.; STEIN, Jeremy C.; Thy Neighbor's Portfolio: Word-of-Mouth Effects in the Holdings and Trades of Money Managers; NBER Working Paper Series; WP 9711, maio de 2003, 47p.

HoTELling, Harold; Stability in Competition, Economic Journal, Vol. 39, No. 153, 1929, pp. 41-57.

Houston, Joel F.; JAMES, Christopher M.; Do relationships have limits? Banking relationships, financial constraints, and investment, Journal of Business, Volume 74(3), 2001, pp. 347-375.

HUMPHREY, David B.; Why do estimates of bank scale economies differ?, Economic Review, Federal Reserve Bank of Richmond, issue Sep, 1990, pp. 38-50.

JaCKLIn, Charles J.; Demand Deposits, Trading Restrictions, and Risk Sharing, in Edward C. Prescott e Neil Wallace (editores), Contractual arrangements for intertemporal trade, Minneapolis, MN : University of Minnesota Press, 1987, pp. 26-47.

JAFFE, Jeffrey F.; Special Information and Insider Trading, Journal of Business, Vol. 47(3), 1974, pp. 410-428.

JEnsen, Michael C.; MecKLIng, William H.; Theory of the firm: Managerial behavior, agency costs and ownership structure, Journal of Financial Economics, volume 3(4), 1976, pp. 305-360.

Johnson, Simon; KWAK, James; 13 Bankers: The Wall Street Takeover and the Next Financial Meltdown, Nova Iorque: Vintage, 2011. 
JUDD, Kenneth L.; Credible Spatial Preemption, RAND Journal of Economics, vol. 16(2), 1985, pp. 153-166.

KAHANE, Yehuda; Capital Adequacy and the Regulation of Financial Intermediaries, Journal of Banking \& Finance, vol. 1(2), 1977, pp. 207-218.

KANE, Edward J.; Interaction of Financial and Regulatory Innovation; American Economic Review, Vol. 78, No. 2, Papers and Proceedings of the One-Hundredth Annual Meeting of the American Economic Association, 1988, pp. 328-334.

KAREKEN, John H.; WALlace, Neil. Deposit Insurance and Bank Regulation: A Partial-Equilibrium Exposition, Journal of Business, vol. 51(3), 1978, pp. 413-438.

KASHYAP, Anil K.; RAJAN, Raghuram; STEIN, Jeremy C.; Banks as Liquidity Providers: An Explanation for the Coexistence of Lending and DepositTaking, Journal of Finance, Volume 57, Issue 1, pages 33-73, February 2002.

KEELEY, Michael C.; Deposit Insurance, Risk, and Market Power in Banking, American Economic Review, 80(5), pp. 1183-1200.

KeETON, William R.; Deposit Insurance and the Deregulation of Deposit Rates, Economic Review, Federal Reserve Bank of Kansas City, edição de abril, 1984, pp. 28-46.

Kim, Daesik; SANTOMERO, Anthony M.; Risk in Banking and Capital Regulation, Journal of Finance, Vol. 43, no. 5, 1988, pp. 1219-1233.

Kim, Moshe; Kristiansen, Eirik G.; VALE, Bent; Endogenous product differentiation in credit markets: What do borrowers pay for?, Journal of Banking \& Finance, volume 29(3), 2005, pp. 681-699.

; VALE, Bent; Non-price strategic behavior: The case of bank branches, International Journal of Industrial Organization, volume 19, 2001, pp. 1583-1602.

KindleBERGER, Charles P.; A Financial History of Western Europe, Oxford: Oxford University Press, 1993, 525p.

KLEIn, Michael A.; A Theory of the Banking Firm, Journal of Money, Credit and Banking, Vol. 3(2), 1971, pp. 205-218.

KrasA, Stefan; VILlamil, Anne P.; Monitoring the Monitor: An Incentive Structure for a Financial Intermediary, Journal of Economic Theory, vol. 57, 1992, pp. 197-221.

KRoES, Neelie; Banks must Refor and Restructure, discurso no Fórum da OCDE "The Crisis and Beyond: For a Stronger, Cleaner, Fairer Economy", Paris, 23 de junho de 2009.

; Time for Banks to Shoulder their Responsibilities, discurso proferido em Conferência organizada pelo Deutsche Bank em Frankfurt, 14 de março de 2009. 
Kydland, Finn E.; PrescotT, Edward C.; Rules Rather than Discretion: The Inconsistency of Optimal Plans; Journal of Political Economy, Vol. 85(3), 1977, pp. 473-492.

LAEVEN, Luc; LEVINE, Ross; Is there a diversification discount in financial conglomerates? Journal of Financial Economics, Volume 85(2), 2007, pp. 331-367.

LANG, Larry; STUlz, René M.; Tobin's q, corporate diversification, and firm performance, Journal of Political Economy, vol. 102(6), 1994, pp. 1248-1280.

LAU, L. J., On identifying the degree of competitiveness from industry price and output data, in Economic Letters, 10 (1982), pp. 93-99.

LEIBENSTEIN, Harvey; Allocative Efficiency vs. "X-Efficiency", American Economic Review, Vol. 56, No. 3, 1966, pp. 392-415.

LELAND, Hayne E.; PYLE, David H.; Informational Asymmetries, Financial Structure, and Financial Intermediation, Journal of Finance, Vol. 32, No. 2, 1977, pp. 371-387.

LERnER, Abba P.; The Concept of Monopoly and the Measurement of Monopoly Power, Review of Economic Studies, 1 (3), 1934, 157-175.

LEVINE, Ross, Financial development and economic growth: views and agenda, in Journal of Economic Literature 35 (1997), pp. 688-726.

RENELT, David, A sensitivity analysis of cross-country growth regressions, in American Economic Review 82:4 (1992), pp. 942-963.

LEVY, Maria Bárbara, A indústria do Rio de Janeiro através de suas sociedades anônimas, Rio de Janeiro, UFRJ, 1994.

História dos bancos comerciais no Brasil (estudo preliminar), Rio de Janeiro, Ibmec, 1972.

LLEWELlYN, David T.; Banking in the 21st Century: The Transformation of an Industry, in M.Edey (Editor), The Future of the Financial System, Sydney, Austrália: Reserve Bank of Autralia, 1996.

LUNDBERG, Eduardo Luis (Org.). Juros e spread bancário no Brasil, in DEPEP - Banco Central do Brasil, 1999.

; Rede de Proteção e Saneamento no Sistema Bancário, in Saddi, Jairo (Org.) - Intervenção e Liquidação Extrajudicial no Sistema Financeiro Nacional - 25 anos da Lei 6.024/74, Textonovo, São Paulo, 1999.

MACEY, Jonathan R.; MILlER, Geoffrey P.; Nondeposit deposits and the future of bank regulation, Michigan Law Review, Vol. 91, novembro de 1992, pp. 237-273.

MACKINLAY, A. Craig, Event studies in economics and finance, Journal of Economic Literature, Vol. XXXV, março de 1997, pp. 13-39. 
MAIA, Geraldo V. S.; Reestruturação Bancária no Brasil: O Caso do PROER, Nota Técnica do Banco Central do Brasil no 38, junho de 2003.

MARques, Cláudia Lima, Contratos bancários em tempos pós-modernos: primeiras reflexões, in RDC 25 (1998), pp.19-38.

Cláusulas abusivas nos contratos imobiliários e bancários, in XI Curso Brasilcon de Direito do Consumidor: Instituto Brasileiro de Política e Direito do Consumidor, 1997.

Contratos bancários no CDC: o paradigma da boa-fé objetiva, in I Seminário Itaú de direito do consumidor, São Paulo, Grupo Itaú, 1997.

MatTheWs, Kent; MuRINDE, Victor; ZHAO, Tianshu; Competitive conditions among the major British banks; Journal of Banking \& Finance, Volume 31, Issue 7, July 2007, pp. 2025-2042.

; THOMPSON, John; The Economics of Banking, 2a Edição Edition, Chichester : John Wiley \& Sons, 2008.

Matutes, Carmen; VIVES, Xavier; Competition for Deposits, Fragility, and Insurance, Journal of Financial Intermediation, vol. 5(2), 1996, pp. 184-216.

MCARTHUR, John W.; SACHS, Jeffrey D.; Institutions and geography: comment on Acemoglu, Johnson and Robinson (2000). NBER Working Paper Series, n. 8.114, 2001.

McGoldrick, Peter J.; GreEnland, Steven J.; Competition Between Banks and Building Societies in the Retailing of Financial Services, British Journal of Management, Volume 3, No 3, pp. 169-179, setembro de 1992.

MCKenZIE, L.; On Equilibrium in Graham's Model of World Trade and Other Competitive System, Econometrica, Vol. 22(2), 1954, pp. 147-161.

Merton, Robert C., An Analytic Derivation of the Cost of Deposit Insurance and Loan Guarantees: An Application of Modern Option Pricing Theory, Journal of Banking \& Finance, vol. 1(1), 1977, pp. 3-11.

Mesquita, Mário; Torós, Mário; Gestão do Banco Central no Pânico de 2008, in Márcio Garcia e Fabio Giambiagi, Risco e Regulação, Rio de Janeiro: Elsevier, 2010, pp. 111-123.

MESTER, Loretta J.; Banking and commerce: a dangerous liaison?, Business Review, Federal Reserve Bank of Philadelphia, maio, 1992, pp. 17-29.

MILLER, Geoffrey P.; On the Obsolescence of Commercial Banking, Journal of Institutional and Theoretical Economics (JITE) / Zeitschrift für die gesamte Staatswissenschaft, Vol. 154, No. 1, The New Institutional Economics Financial Institutions in Transition: Banks and Financial Markets, março de 1998, pp. 61-73.

MinistÉRIO DA FAZENDA, Conheça o Tesouro Nacional, Brasília, 2011. 
Mishkin, Frederic S. The Economics of Money, Banking, and Financial Markets, 7a edição, Boston: Pearson-Addison Wesley, 2004.

Is monetary policy effective during financial crises? , in American Economic Review 99:2 (2009), pp. 573-577.

; How big a problem is too big to fail? A review of Gary Stern and Ron Feldman's Too Big to Fail: The Hazards of Bank Bailouts, Journal of Economic Literature, Vol. XLIV, dezembro de 2006, pp. 988-1004, especialmente pp. 989-990.

Mitchell, Douglas W., Interest-Bearing Checking Accounts and Macro Policy, Tese de Doutorado, Princeton University, 1978, pp. 3-4.

MonTI, Mario; Deposit, credit, and interest rate determination under alternative bank objectives, in Karl Shell and Giorgio P. Szego (editores), Mathematical Methods in Investment and Finance, Amsterdam: NorthHolland, 1972, pp. 431-454.

Morgan, Thomas D., Cases and materials on modern antitrust law and its origins, $4^{\text {a }}$ Ed, St. Paul, West, 2009.

MotтA, Massimo, Competition policy: theory and practice, Cambridge, Cambridge University, 2004.

Mullineux, Andy W.; International Banking and Financial Systems: A Comparison, Londres: Graham and Trotman, 1987, 243 p.

Musacchio, A., Law and finance in historical perspective : politics, bankruptcy law, and corporate governance in Brasil, 1850-2002, Tese de Doutorado, Stanford University, 2005.

NAKANE, Márcio L., A test of competition in Brazilian banking, in Banco Central do Brasil Working Paper Series 12 (2001).

Concorrência e spread bancário: uma revisão da evidência para o

Brasil. in Lundberg, Eduardo L. (Org.), Economia bancária e crédito: avaliação de quatro anos do projeto juros e spread bancário, Brasília, Banco Central do Brasil, 2003, pp. 58-67.

Nathan, Alli, NeAVE, Edwin H., Competition and contestability in Canada's financial system: empirical results, Canadian Journal of Economics 22 (1989), pp. 576-594.

NORTH, Douglass C., Institutions, institutional change and economic performance, New York, Cambridge University, 1990.

Noyes, Alexander D.; Forty Years of American Finance, Nova Iorque, Londres: G. P. Putnam's and Sons, 1909, 418p.

OCDE, Policy Framework for Effective and Efficient Financial Regulation, Paris, 2010. 
, Building an institutional framework for regulatory impact analysis (RIA): guidance for policy makers, Regulatory Policy Division, Directorate for Public Governance and Territorial Development, Paris, version $1.1,2008$.

Introductory handbook for undertaking regulatory impact analysis (RIA), version 1.0, outubro de 2008.

, Recommendation of the council of the OECD on improving the quality of government regulation, Paris, 1995.

OFFICE OF FAIR TRADING, Anticipated acquisition by Lloyds TSB plc of HBOS plc, Report to the Secretary of State for Business Enterprise and Regulatory Reform, 24 de outubro de 2008.

O'HARA, Maureen; SHAw, Wayne; Deposit Insurance and Wealth Effects: The Value of Being "Too Big to Fail", The Journal of Finance, Vol. 45(5), 1990, pp. 1587-1600.

OLIVEIRA, Gesner, Defesa da concorrência e regulação no setor bancário, in Campilongo, CF, Rocha, JPC e Mattos, PT, Concorrência e regulação no sistema financeiro, São Paulo, Max Limonad, 2002.

OLSON, Mancur; The Logic of Collective Action: Public Goods and the Theory of Groups, Cambridge, MA: Harvard University Press, 1965.

PANZAR, J., Rosse, James, Testing for "monopoly" equilibrium, in Journal of Industrial Economics 35:4 (1987), pp. 443-456.

Peltzman, Sam, The gains and losses from industrial concentration, in Journal of Law and Economics 20 (1977), pp. 229-263.

; Toward a more general theory of regulation, Journal of Law and

Economics, Chicago, v.19, 1976.

PENAS, María F.; UnAL, Haluk; Gains in bank mergers: Evidence from the bond markets, Journal of Financial Economics, Volume 74(1), 2004, pp. 149179 .

PETERSEN, Mitchell A.; RAJAN, Raghuram G.; Does Distance Still Matter? The Information Revolution in Small Business Lending, Journal of Finance, vol. 57(6), 2002, pp. 2533-2570.

PetTERINI, Francis C, JoRge Neto, Paulo de M., Competição bancária no Brasil após o plano Real, in VIII Encontro Regional de Economia do Nordeste, Fortaleza, Julho de 2003.

Poser, Norman S.; Broker Dealer Law and Regulation, Nova Iorque: Aspen, 2001.

Postlewaite, Andrew; VIVES, Xavier; Bank Runs as an Equilibrium Phenomenon, Journal of Political Economy, Vol. 95(3), 1987, pp. 485-491. 
Prisman, Eliezer Z.; Slovin, Myron B.; SushKA, Marie E.; A General Model of the Banking Firm under Conditions of Monopoly, Uncertainty, and Recourse, Journal of Monetary Economics, Volume 17(2), 1986, pp. 293-304.

PYLE, David H.; On the Theory of Financial Intermediation, Journal of Finance, Vol. 26(3), 1971, pp. 737-747.

RADECKI, Lawrence J.; The Expanding Geographic Reach of Retail Banking Markets, FRBNY Economic Policy Review, junho de 1998, pp. 15-34.

RAJAN, Raghuram G.; Is there a future in banking? Towards a new theory of the commercial bank, Northwestern University e University of Chicago, 1996, 33p.

RAJAN, Raghuram G.; ZINGALES, Luigi; Saving capitalism from the capitalists, Princeton: Princeton University Press, 2003, pp. 5-6.

ReInhart, Carmem M.; Rogoff, Kenneth S.; This Time is Different: Eight Centuries of Financial Folly, Princeton: Princeton University Press, 2009.

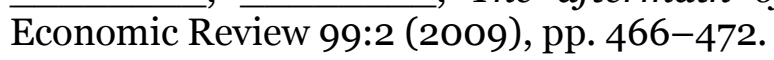

The aftermath of financial crises, in American

RHOADES, Stephen A.; Summary of Merger Performance Studies in Banking, 1980-93, and an Assessment of the Operating Performance and Event Study Methodologies, 8o Federal Reserve Bulletin, pp. 589, 1994.

RIBEIRO, Ivan C., Regulatory impact analysis and cost benefit analysis: what is different across the sea, Revista do Direito Público da Economia, Ano 8, no 32, Out-Dec. 2010, pp. 73-87.

; Cost Benefit Analysis in Financial Regulation: First Lessons from the 2008 Crisis, Revista de Direito Bancário e do Mercado de Capitais, Ano 13, Vol. 50, Out-Dec 2010, pp. 47-75.

; Revisão bibliográfica e fundamentação da metodologia: consultoria para desenvolvimento de metodologia para análise de custos e benefícios da regulação do mercado de valores mobiliários, relatório preparado para a Comissão de Valores Mobiliários, 2010, 69p.

; Manual para a Análise Econométrica de Custos e Benefícios da Regulação, mimeo não publicado, 2010, consultoria desenvolvida para a Comissão de Valores Mobiliários através do PNUD (Programa das Nações Unidas para o Desenvolvimento, órgão subsidiário da Organização das Nações Unidas, através do Contrato no 2010/000837.

; Concorrência bancária e determinantes institucionais da taxa de juros: uma análise empírica de séries de tempo in Revista do IBRAC 13:1 (2006), pp. 139-168.

; A Influência da Insegurança Jurídica sobre as taxas de juros na alienação fiduciária, in Cavalcanti, Nicolau da Rocha, Ribeiro, Ivan César, 
Crédito imobiliário, São Paulo, Instituto Tendências de Direito e Economia, 2005 .

; Contratos Relacionais e Teoria da Firma, dissertação de mestrado apresentado à Universidade de São Paulo, 2005.

; ForTt, Sarah E.; MitZENMACHER, Eric, PEAR, Matthew; Assessing

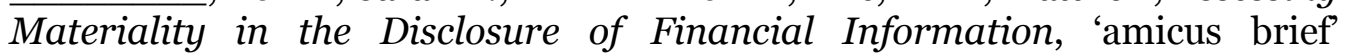
apresentado a Securities and Exchange Comission (SEC), 2009.

RICH, Jonathan M., SCRIVEN, Thomas G.; Bank Consolidation Caused by the Financial Crisis: How Should the Antitrust Division Review 'Shotgun Marriages'?, The Antitrust Source, dezembro de 2008, pp. 1-8.

RISKBANK, FUSÕES \& AQUISIÇÕES (desde 1998 até 2012), 2012, Relatório disponível em http://www.riskbank.com.br, consultado em 09.05.2012.

Rochet, Jean-Charles, Capital Requirements and the Behaviour of Commercial Banks, European Economic Review, vol. 36(5), 1992, pp. 11371170 .

Rodrigues, Eduardo A. S., TAKEDA, Tony, ARAÚJo, Aloísio P., Qual o impacto das garantias reais nas taxas de juros de empréstimo bancário no Brasil? uma breve avaliação com base nos dados do SCR, in Banco Central do Brasil - Seminário de Economia Bancária e Crédito, Brasília, dezembro de 2004.

ROTEMBERG, Julio J., SALONER, Garth, The relative rigidity of monopoly pricing, in American Economic Review 77 (1987), pp. 917-926.

SACHS, Jeffrey D.; Tropical underdevelopment, NBER Working Paper 8119, 2001.

SADDI, Jairo, Concentração no Sistema Financeiro Nacional, in RDE 26 (1997).

SAES, Flávio A. M., Crédito e bancos no desenvolvimento da economia paulista, 1850-1930, São Paulo, IPE/USP, 1986.

SALOMÃo FILHo, Calixto, Monopólio colonial e subdesenvolvimento, in Direitos humanos, democracia e república - homenagem à Fábio Konder Comparato, São Paulo, Quartier Latin, 2009.

; Direito como instrumento de transformação social e econômica, in RDPE 1 (2003), pp. 15-44.

; Direito concorrencial: as condutas, São Paulo, Malheiros, 2003.

; Direito concorrencial: as estruturas, São Paulo, Malheiros, $2^{\mathrm{a}} \mathrm{Ed}$,

2002.

; (Org.), Regulação e desenvolvimento, São Paulo, Malheiros, 2002.

Malheiros, 2002.

; Regulação e concorrência - estudos e pareceres, São Paulo, 
; Regulação e antitruste: fronteiras e formas de interação no setor financeiro, in Campilongo, CF, Rocha, JPC e Mattos, PT, Concorrência e regulação no sistema financeiro, São Paulo, Max Limonad, 2002.

; Regulação da atividade econômica: princípios e fundamentos jurídicos, São Paulo, Malheiros, 2001.

; Apontamentos para formulação de uma teoria jurídica dos cartéis, in RDM 121 (2001), pp. 18-29.

(1999), pp. 51-62.

; Condutas Anticoncorrenciais no Setor Bancário, in RDM 114

; Direito empresarial público, in RDM 112 (1998), pp. 9-18.

; Atuação estatal e ilícito antitruste, in RDM 106 (1997), pp. 35-47.

SAMUELSON, Paul; An Exact Consumption-Loan Model of Interest with or without the Social Contrivance of Money, Journal of Political Economy, vol. 66, 1958, pp. 467-482.

SCARTEZzINI, Jorge T. F., Conflito de atribuições - noções gerais e aspectos relevantes, in RIASP 5:3 (2000).

SCHAECK, Klaus, CIHAK, Martin, Wolfe, Simon, Are competitive banking systems more stable?, Journal of Money, Credit and Banking 41:4, 2009, pp. 711-734.

SCHMALENSEE, Richard; Entry deterrence in the ready-to-eat breakfast cereal industry, Bell Journal of Economics, 1978, vol. 9(2), pp. 305-327.

SCHMIDT, Reinhard H.; The Future of Banking in Europe, FinancialMarkets and Portfolio Management, vol. 15, no 4, 2001, pp. 429-449, especialmente p. 430.

; The Future of Banking in Europe, Working Paper Series: Finance and Accounting 72, Department of Finance, Goethe University Frankfurt am Main, 2001.

; Hackethal, Andreas; TyRell, Marcel; Disintermediation and the Role of Banks, Journal of Financial Intermediation, vol. 8, 1999, pp. 36-67, especialmente pp. 50-51.

SCHOLES, Myron S., The market for securities: substitution versus price pressure and the effects of information share prices, Journal of Business, vol. 45, abril de 1972, pp. 179-211.

SchulZ, John, The financial crisis of abolition, New Haven, Yale University, 2008.

; A Crise Financeira da Abolição, São Paulo, Edusp, 1996, 168p.

SCHWERT, G. William, Using financial data to measure effects of regulation, Journal of Law and Economics, vol. 24, abril de 1981, pp. 121-158, especialmente pp. 132-134. 
SECRETARIA DE Política ECONÔMICA - Ministério da Fazenda, Reformas microeconômicas e crescimento de longo prazo. Brasília, Dezembro de 2004.

SHAFFER, Sherrill; A Test of Competition in Canadian Banking; Journal of Money, Credit and Banking, Vol. 25, No. 1, 1993, pp. 49-61.

SHUll, Bernard, HANWECK, Gerald A., Bank mergers in a deregulated environment: promise and peril, Westport, Quorum Books, 2001.

SifFERT FILHO, Nelson; Governança corporativa: padrões internacionais e evidências empíricas no Brasil nos anos 90. Revista do BNDES, n. 9, 1998.

Simon, Leo K.; Basic Timing Games, Economics Working Papers 8745, University of California at Berkeley, 1987.

; STINCHCOMBe, Maxwell B; Extensive Form Games in Continuous

Time: Pure Strategies, Econometrica, vol. 57(5), 1989, pp. 1171-1214.

Slovin, Myron B.; SushKa, Marie E.; PoloncheK, John A.; An analysis of contagion and competitive effects at commercial banks, Journal of Financial Economics, Volume 54(2), 1999, pp. 197-225.

SNELL, Bradford C.; Annual style change in the automobile industry as an unfair method of competition - parte 1, volume 4(1), 1970-1971, pp. 67-94.

; Annual style change in the automobile industry as an unfair method of competition - parte 2, volume 4(2), 1970-1971, pp. 55-92.

SOUZA Franco, Bernardo de, Os bancos do Brasil: sua história, defeitos de organização atual e reforma do sistema bancário, Rio de Janeiro, Typ. Nacional, 1848.

SPENCE, Michael; Job Market Signaling, Quarterly Journal of Economics, 87(3), 1973, pp. 355-374).

SPILLER, Pablo T.; FAVARO, Edgardo; The effects of entry regulation on oligopolistic interaction - the Uruguayan banking sector, in Rand Journal of Economics 15(2), 1984, pp. 244-254.

SPRAGUE, Irvine H.; Bailout, Nova Iorque: Basic Books, 1986.

SRINIVASAN, Aruna; Are there cost savings from bank mergers?, Economic Review, Federal Reserve Bank of Atlanta, issue Mar, 1992, pp. 17-28.

Steen, F., SAlvanes, K. G., Testing for market power using a dynamic oligopoly model, in International Journal of Industrial Organization 17 (1999), pp. 147-177.

STEIN, Jeremy C.; Information production and capital allocation: decentralized vs. hierarchical firms, Journal of Finance 57:5 (2002), pp. 18911921.

STIGLER, George J.; The theory of economic regulation, Bell Journal of Economic and Management Science, New York, v. 2, n. 1, p. 1-21, 1971. 
STIROH, Kevin J.; RuMble, Adrienne; The dark side of diversification: The case of US financial holding companies, Journal of Banking \& Finance, Volume 30(8), 2006, pp. 2131-2161.

SUAREZ, Javier; Closure Rules, Market Power and Risk-Taking in a Dynamic Model of Bank Behavior, LSE Financial Markets Group Discussion Paper 196, 1994.

Sullivan, Edward Thomas, HovenKamp, Herbert, Antitrust law, policy, and procedure: cases, materials, problems, $3^{\mathrm{a}} \mathrm{Ed}$, Charlottesville, Michie Co., 1994.

SUPREMO TRIBUNAL FEDERAL, A Justiça em números - indicadores estatísticos do Poder Judiciário Brasileiro, in A Justiça ..., Brasília, Supremo Tribunal Federal, 12 e 13 de maio de 2005.

SZTAJN, Rachel. Regulação e concorrência no sistema financeiro, in Campilongo, CF, Rocha, JPC e Mattos, PT, Concorrência e regulação no sistema financeiro, São Paulo, Max Limonad, 2002.

TABAK, Benjamin M., GUERrA, Solange M G, Lima, Eduardo J. A., Chang, Eui J., The stability-concentration relationship in the Brazilian banking system, Banco Central - Working Papers Series 145 (2007).

; Fazio, Dimas M.; CajueIRo, Daniel O.; Profit, Cost and Scale Efficiency for Latin American Banks: Concentration-Performance Relationship; Banco Central do Brasil - Working Papers Series, WP244, maio de 2011.

TAVAres, Martus A. R.; CARvalheiro, Nelson; O setor bancário brasileiro: Alguns aspectos do crescimento e da concentração, São Paulo: FIPE/USP, 1985, 208p, especialmente seção 2.2, pp. 31-33.

THEIL, H., Principles of econometrics, Nova Iorque: Wiley, 1971, pp. 122-123.

TiROLE, J., Financial crises, liquidity, and the international monetary system, Princeton, Princeton University, 2002.

1988.

The theory of industrial organization, Cambridge, MIT Press,

ToBIN, James; Financial Innovation and Deregulation in Perspective, Cowles Foundation Paper 635, Bank of Japan Monetary and Economic Studies, vol. 3, no 2, 1985, pp. 19-29.

TodD, Walker F.; THOMson, James B.; An insider's view of the political economy of the too big to fail doctrine, Working Paper 9017, Federal Reserve Bank of Cleveland, 1990.

TonookA, Eduardo Kiyoshi, Koyama, Sérgio Mikio, Taxa de juros e concentração bancária no Brasil, Trabalho para discussão 62, Banco Central do Brasil (2003). 
TOWNSEND, Robert M., Optimal contracts and competitive markets with costly state verification, Journal of Economic Theory, vol. 21(2), 1979, pp. 265-293.

TREASURY COMMITTEE, Competition \& choice in retail banking, relatório publicado em 02/04/2011.

Treasury DePartment, The Department of the Treasury Blueprint for a Modernized Financial Regulatory Structure, março de 2008.

Troster, Roberto L.; Regulamentação Bancária Brasileira: Situação Atual e Perspectivas, in Anais do Seminário Internacional sobre Regulação e Defesa da Concorrência no Setor Bancário, CADE/ASBACE, março de 1999, especialmente pp. 344-345.

; Os bancos são diferentes?, in Campilongo, CF, Rocha, JPC e

Mattos, PT, Concorrência e regulação no sistema financeiro, São Paulo, Max Limonad, 2002.

U.S. Department of Justice e Federal Trade Commission, Horizontal Merger Guidelines, revisão de 1997.

U.S. GOVERnMENT ACCOUNTABILITY OFFICE, Financial Regulation: A Framework for Crafting and Assessing Proposals to Modernize the Outdated U.S. Financial Regulatory System, audiência no Congressional Oversight Panel, Jan. 8, 2009.

UHDE, André, HeImeshofF, Ulrich, Consolidation in banking and financial stability in Europe: empirical evidence, in Journal of Banking \& Finance 33 (2009), pp. 1299-1311.

UNCTAD - United Nations Conference on TRAde AND Development, UNCTAD Guidebook on Competition Systems, Nova Iorque e Genebra, 2007, $167 \mathrm{p}$.

VAle, Bent; The Dual Role of Demand Deposits under Asymmetric Information, The Scandinavian Journal of Economics , Vol. 95, No. 1 (Mar., 1993), pp. 77-95.

VAN LeUvensteiJn, Michie; SøRensen, Christoffer K.; BIKKeR, Jacob A.; VAN RIXTELD, Adrian A.R.J.M.; Impact of Bank Competition on the Interest Rate Pass-through in the Euro Area, Applied Economics, Volume 45, Issue 11, 2013, pp. 1359-1380.

VAZ, Isabel. Fundamentos constitucionais da livre concorrência no setor bancário, in Campilongo, CF, Rocha, JPC e Mattos, PT, Concorrência e regulação no sistema financeiro, São Paulo, Max Limonad, 2002.

VerçOSA, Haroldo Malheiros Duclerc, A recepção do drop down no direito brasileiro in RDM 125 (2002), pp. 41-47.

A reformulação da Lei 6.024, de 13.3.1974 in RDM 41 (1981), pp.

29-39. 
Bancos Centrais no direito comparado: o Sistema Financeiro Nacional e o Banco Central do Brasil (o regime vigente e as propostas de reformulação, São Paulo, Malheiros, 2005.

Conselho Monetário Nacional: competência reguladora - as auditorias independentes, in RDM 124 (2002), pp. 208-214.

, Notas sobre o regime jurídico das ofertas ao público de produtos, serviços e valores mobiliários no direito brasileiro: um caso de complementação da proteção de consumidores e investidores, in RDM 105 (1997), pp. 74-83.

O conceito de reservas bancárias e as características do mútuo e do depósito bancário in RDM 110 (1998), pp. 207-211.

VICKERS, John, The Financial Crisis and Competition Policy: Some Economics, in GCP - The Online Magazine for Global Competition Policy release 1, Dezembro de 2008.

VIVANTE, Cesare. Os comerciantes, Vol. 1, $5^{\text {a }}$ Ed, Milão, Casa Editrice Dottor Francesco Vallardi, 1934, tradução de Verçosa, Haroldo Malheiros Duclerc, in RDM 102 (1996), pp. 134-138.

VIVES, Xavier, Oligopoly pricing: old ideas and new tools, Cambridge, MIT Press, 2000.

; Bank Competition and European Integration, in Alberto G. Giovannini, Colin Mayer (Editores), European Financial Integration, Cambridge: Cambridge University Press, 1992.

VoIGT, Stefan; The Effects of Competition Policy on Development - CrossCountry Evidence Using Four New Indicators, Journal of Development Studies, Volume 45(8), 2009.

VON THADDEN, Ernst-Ludwig; Asymmetric information, banklending and implicit contracts: the winner's curse, Finance Research Letters, Volume 1, Issue 1, March 2004, pp. 11-23.

; The term-structure of investment and the banks' insurance function, European Economic Review, Volume 41, Issue 7, Julho 1997, pp. 1355-1374.

VUONG, Quang H., Likelihood ratio tests for model selection and non-nested hypotheses, in Econometrica 57 (1989), pp. 307-333.

WAGNER, Wolf; Diversification at financial institutions and systemic crises, Journal of Financial Intermediation, Volume 19(3), 2010, pp. 373-386.

WALD, Arnoldo, Conflito de atribuições: a recente evolução do conflito de atribuições, in RTJERJ 15 (1993).

Estudos e pareceres de direito comercial: problemas comerciais e fiscais da empresa contemporânea, São Paulo, Revista dos Tribunais, 1979. 
, O novo direito monetário, $2^{\mathrm{a}} \mathrm{Ed}$, Malheiros, 2002.

WALL, Larry D.; A Plan for Reducing Future Deposit Insurance Losses: Putable Subordinated Debt, Economic Review, Federal Reserve Bank of Atlanta, vol. 74, 1989, pp. 2-17.

Williamson, O. E.; The Economic Institutions of Capitalism: Firms, Markets, Relational Contracts, New York, The Free Press, 1985.

; The Mechanisms of Governance, New York, Oxford University, 1996.

ZARDHOOHI, Asghar; KOLARI, James W.; Branch office economies of scale and scope: Evidence from savings banks in Finland, Journal of Banking and Finance, vol. 18, 1994, pp. 421-432.

\section{Periódicos}

BACHELOR, Lisa, HBOS: the issue explained, in The Guardian, disponível [online] <http://www.guardian.co.uk/money/2001/may/o4/personalfinancenews2>, [04.05.2001].

PARKER, George; CROFT, Jane; TAIT, Nikki; EU ruling threatens Lloyds' branches, publicada também pelo Financial Times, 25 de setembro de 2009, disponível em http://www.ft.com/intl/cms/s/o/b633cf86-aao8-11de-a3ceo0144feabdco.html\#axzz2725bpaLo, consultado em 10/10/2009.

SUPERIOR TRIBUNAL DE JUSTIÇA, STJ começa a definir a quem compete tratar da aquisição de um banco por outro: se Cade ou Bacen, disponível [on-line] in <http://www.stj.gov.br/portal_stj/publicacao/engine.wsp?tmp.area=398\& tmp.texto=94119\# $>$ [07.10.2009].

TAIT, Nikki; JENKIns, Patrick; CROFT, Jane; Kroes warns of forced Lloyds asset sales, Financial Times, 30 de junho de 2009, disponível em http://www.ft.com/intl/cms/s/o/4d122398-6586-11de-8e34-

oo144feabdco.html\#axzz2725bpaLo, consultado em 10/10/2009. 


\section{Casos}

Basic v. Levinson, 485 U.S. 224 (1988).

17 CFR 275, Political contributions by certain investment advisers: final rule, in Federal Register n ${ }^{\circ}$ 75, pp. 41.018-41.071, 14 de julho de 2010.

AC 08012.0011303/2008-96, Banco Itaú S.A. e Unibanco, com decisão em $18 / 08 / 2010$

AC 08012.011736/2008-41, Banco do Brasil S.A. e Banco Nossa Caixa S.A., decidido em 04/08/2010.

474 US 361, Board of Governors of the Federal Reserve System V. Dimension Financial Corporation.

FTC v. Kellogg Company (99 FTC 8)

ADI 2591/DF, rel. orig. Min. Carlos Velloso, rel. p/ o acórdão Min. Eros Grau, STF.

Processo CVM RJ-2003/6098 - Acórdão/CRSFN 7877/o6, rel. Cons. Flávio Maia Fernandes dos Santos.

Caso - Comissão Europeia, Diretoria Geral de Concorrência, Comissão Europeia, State Aid Case N428/2009 - Reino Unido.

Caso - Comissão Europeia, Diretoria Geral de Concorrência, Comissão Europeia, State Aid Case N422/2009 e N621/2009 - Reino Unido.

Caso - Comissão Europeia, Diretoria Geral de Concorrência, Comissão Europeia, State Aid Case N244/2009.

Caso - Comissão Europeia, Diretoria Geral de Concorrência, Comissão Europeia, State Aid Case C10/2009 (antigo N138/2009), OJ C158, 11/07/2009

Caso - Comissão Europeia, Diretoria Geral de Concorrência, Comissão Europeia, State Aid Case 528/2008, ING, de 23 de dezembro de 2008

C14/2008 (Northern Rock).

C18/2009 (KBC)

N531/2009 (West LB)

Citizen Publishing Co. V. United States, 394 U.S. 131, 136-138 (1969)

United States v. General Dynamics Corp., 415 U.S. 485 (1974)

FTC v. Arch Coal, Inc, 329 F. Suoo. $2 d$ 109, 146-47 (D.D.C. 2004)

United States v. UPM-Kymmene Oyj, 2003-2 Trade Cas. (CCH) parágrafos 74, 101 (N.D. Ill. 2003))).

United States v. Phila. Nat'l Bank, 374 U.S. 321 (1963).

United States v. Third Nat'l Bank, 390 U.S. 171 (1968). 
AMERICAN BAR ASSOCIATION, Section on Antitrust Law, Federal statutory exemptions from antitrust law, Monograph 24, 2007, p. 50 e nt 124.

Resp. 109.421-8, STJ

RE 664189, STF

Ato de Concentração número 08012.006762/2000-09, tendo como requerentes o Banco Finasa de Investimento S/A, a Brasmetal Indústria S/A e a Zurich Participações e Representações.

Merger Action Group v Secretary of State for Business, Enterprise and Regulatory Reform, [2008] CAT 36.

\section{Regulações}

12 CFR 252.

12 U.S.C. Sec. $1823(\mathrm{c})(4)(\mathrm{A})$

Resolução 4087

Resoluções $\mathrm{n}^{0}$ 3.251, de 16 de dezembro de 2004, $\mathrm{n}^{0} 3.400$, de 6 de setembro de 2006, e $\mathrm{n}^{0} 3.656$, de 17 de dezembro de 2008

Resolução 2.197 de 31.08.1995.

Guia para Análise de Atos de Concentração envolvendo instituições financeiras, divulgado através do Comunicado 22.366 de 27 de abril de 2012.

Circular 3179, de 26 de fevereiro de 2003

Lei 7495/86

Lei 7.730 de 31 de janeiro de 1989

12 CFR 204.

12 CFR 217.

12 CFR 225

CVM no 409, de 18 de agosto de 2004,

Treaty Functioning European Union (TFEU), Tratado de Roma.

Bank Merger Act e pelo Bank Holding Company Act , 12 U.S.C. §§ 1828(c), 1849(b).

Regulação Q (12 CFR 217) 